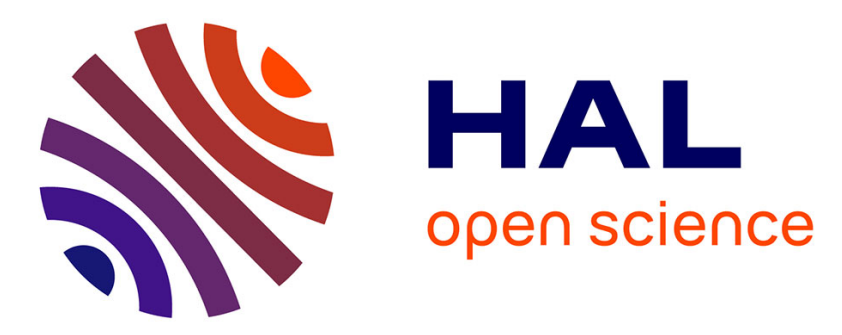

\title{
Uniform observability analysis for structured bilinear systems: A graph-theoretic approach
}

\author{
Taha Boukhobza, Frédéric Hamelin, Dominique Sauter
}

\section{To cite this version:}

Taha Boukhobza, Frédéric Hamelin, Dominique Sauter. Uniform observability analysis for structured bilinear systems: A graph-theoretic approach. European Journal of Control, 2006, 12 (5), pp.505-522. hal-00120933

\section{HAL Id: hal-00120933 https://hal.science/hal-00120933}

Submitted on 18 Dec 2006

HAL is a multi-disciplinary open access archive for the deposit and dissemination of scientific research documents, whether they are published or not. The documents may come from teaching and research institutions in France or abroad, or from public or private research centers.
L'archive ouverte pluridisciplinaire HAL, est destinée au dépôt et à la diffusion de documents scientifiques de niveau recherche, publiés ou non, émanant des établissements d'enseignement et de recherche français ou étrangers, des laboratoires publics ou privés. 


\title{
Uniform observability analysis for structured bilinear systems: A graph-theoretic approach
}

\author{
T. Boukhobza, F. Hamelin and D. Sauter \\ Research Centre in Automatic Control (CRAN - CNRS UMR 7039), \\ Henri Poincaré University, \\ BP 239, 54506 Vandœuvre Cedex, Nancy, France, \\ Phone: 33383684 464, Fax: 33383684 462, \\ email: taha.boukhobza@cran.uhp-nancy.fr
}

April 7, 2006

\begin{abstract}
This paper deals with the analysis of generic uniform observability for structured bilinear systems. The proposed method is based on a graph-theoretic approach and assumes only the knowledge of the system's structure. We express, in graphical terms two conditions, the first one is necessary and the second one is sufficient, to check whether or not they are generically uniformly observable. These conditions are quite easy to verify because they are based on comparisons of integers and on finding edges and subgraphs in a digraph. Therefore, our approach is very well suited to study large scale and/or uncertain systems.
\end{abstract}

Keywords: Structured bilinear systems, uniform observability, graph theory, generic properties.

\section{Introduction}

For nonlinear systems, the observability notion is based on the concept of indistinguishability of two initial states for every input. In fact, it results from $[3,13,15,21,24]$ that a nonlinear system is observable if it can be found an input such that any pair of initial states are distinguishable by observation of the corresponding output. The distinguishability of two initial states is thus depending on the input applied to the system. In this respect, for nonlinear systems, the notion of universal input is defined $[13,21]$ as an input for which every pair of initial states can be distinguished by observation of the output. A system, for which every input is universal is said uniformly observable knowing that we are interested in the inputs which take only real values. We call these 
inputs admissible inputs. In other words, a system is uniformly observable when we can express its whole state components in function of the measured variables and their derivatives for all the admissible input values. The analysis of the uniform observability is important since it informs us on the possible alteration of the state distinguishability for some input values. This information is useful obviously for the design of nonlinear observers and also for control, fault detection and isolation and reconfiguration schemes which both use the estimated state variables.

Many studies are dedicated to the uniform observability $[10,11,24]$. In most cases, either the authors are interested only in the single output case or they provide geometric trivial conditions to achieve uniform observability. These conditions are mainly difficult to apply to large scale systems because they are based on the computation of the rank of the observability matrix. Note that some studies assume that the system can be transformed into some triangular form which ensures the uniform observability and which is used in the design of nonlinear observers $[2,7,14,19]$. Finally, in the single output case, a triangular canonical form for uniformly observable systems is provided [9].

In this context, this paper is dedicated to the characterization of the uniform observability of a particular class of nonlinear systems. According to their applicative interest and their intrinsic simplicity, we focus on multi-output bilinear systems. This class of systems, whose dynamics are jointly linear in the state and the input was introduced in control theory in the 1960's. Industrial process control, economics and biology (switched circuits, mechanical brakes, controlled suspension systems, immunological systems, population growth, enzyme kinetics, ... ) provide examples of bilinear systems (BLS). Furthermore, this kind of systems are simpler and better understood than most other nonlinear systems. Finally, the usual linearization of a nonlinear control system near an equilibrium point can be improved by using a bilinear approximation. For these reasons many works deal with BLS.

Generally, BLS are studied in connection with the theories of time-variant linear systems and matrix Lie groups $[4,16,20]$. However, the use of such tools assumes the exact knowledge of the state space matrices characterizing the system's model. In many modeling problems, these matrices have a number of fixed zero entries determined by the physical laws while the remaining entries are not known precisely. This is also true during the system conception stage. To study the properties of these systems in spite of the poor knowledge we have on them, the idea is that we only keep the zero/nonzero entries in the state space matrices. Thus, we consider models where the fixed zeros are conserved while the nonzero entries are replaced by free parameters. There is a huge amount of interesting works in the literature using this kind of models called structured 
models. These models are useful to describe the class of systems having the same structure and they capture most of the structural available information from physical laws. Moreover, their study requires a low computational burden which allows to deal with large scale systems. Because of these features, we think that structured systems are suited to study the uniform observability and subsequently this paper deals with this kind of systems.

Many studies on structured systems are related to the graph-theoretic approach. Until now, this approach is principally dedicated to linear systems for which structural properties, such as controllability, observability, solvability of several classical control problems including disturbance rejection, input-output decoupling, fault detection and isolation, are studied. Survey paper [8] reviews the most significant results in this area. From these works, it results that the graph-theoretic approach provides simple and elegant solutions and so is very well suited to analyse large scale or/and uncertain systems. Unfortunately, few works based on graph-theoretic methods deal with nonlinear systems. Among them, [22] gives sufficient conditions to fulfill the observability of bilinear systems and more recently, [1] provides sufficient conditions for checking the uniform observability of nonlinear systems which are preliminarily put in a canonical form and where the nonlinearities satisfy Lipschitz conditions.

The aim of this paper is to provide a necessary and a sufficient conditions to achieve the uniform observability of multi-output structured bilinear systems (SBLS). The study of this property is based on a graphic representation, which does not necessitate any preliminary computation. Comparatively with the results of [9] which enounce necessary and sufficient conditions to the uniform observability of single output nonlinear systems, our results concern only a particular class of nonlinear system, but for multi-output and structured systems. In the single output case, our conditions are also necessary and sufficient. Moreover, in [24], the author give a geometric necessary and sufficient criterion to the uniform observability of a multi-output bilinear system. This criterion is not a structural one i.e. depends on the parameters of the system, is quite trivial as it assesses that a system is uniformly observable if and only if the rank of the observability matrix is equal to the dimension of the state for every input. This criterion is difficult to verify especially in the case of large scale or uncertain systems. Starting from an equivalent criterion to the one given in [24] and using original mathematical tools, we give two conditions (one necessary and the other sufficient) to the uniform observability of structured bilinear systems which are more "computable" and which depends only on the structure of the systems and not on its parameters. The main interest of the proposed conditions are their easy computability and the fact that they allows to handle system's structure. 
The paper is organised as follows: after section 2, which is devoted to the problem formulation, a digraph representation of SBLS is given in section 3. The main result of the paper is provided in section 4 and is illustrated with an example in section 5. Finally, some concluding remarks are made.

\section{Problem statement}

In this paper, we are interested in the uniform observability of SBLS:

$$
\left(\Sigma_{\Lambda}\right):\left\{\begin{array}{l}
\dot{x}=A^{\lambda} x+\sum_{i=1}^{m} u_{i} B_{i}^{\lambda} x \\
y=C^{\lambda} x \\
z \stackrel{\text { def }}{=} \dot{x}-\sum_{i=1}^{m} u_{i} B_{i}^{\lambda} x
\end{array}\right.
$$

where $x \in \mathbb{R}^{n}, u \in \mathbb{R}^{m}$ and $y \in \mathbb{R}^{p}$ are respectively the state vector, the input vector and the output vector. New variable $z \in \mathbb{R}^{n}$ called dynamic variable, is introduced in this paper. $A^{\lambda} \in \mathbb{R}^{n \times n}, B_{i}^{\lambda} \in$ $\mathbb{R}^{n \times n}$ for $i=1, \ldots, m$ and $C^{\lambda} \in \mathbb{R}^{p \times n}$ represent matrices whose elements are either fixed to zero or assumed to be free non-zero parameters. The vector of these parameters is $\Lambda=\left(\lambda_{1}, \lambda_{2}, \ldots, \lambda_{h}\right)^{T}$. If all the non-zero parameters $\lambda_{i}$ are fixed, we obtain an admissible realization of structured system $\left(\Sigma_{\Lambda}\right)$. Theoretic properties of each realization can be studied according to the values of $\lambda_{i}$.

We say that a property is true generically if it is true for almost all realizations of the system or equivalently for almost all parameter values [6]. Here "for almost all parameter values" is to be understood [8] as "for all parameter values $\left(\Lambda \in \mathbb{R}^{h}\right)$ except for those in some proper algebraic variety in the parameter space ". The proper algebraic variety for which the property is not true is the zero set of some nontrivial polynomial with real coefficients in the $h$ parameters of the system, which can be written down explicitly. Recall that a proper algebraic variety is an algebraic variety which has Lebesgue measure zero. A property which is true for almost all parameters values is also said to be true generically or to be a generic property for the considered structured system. In order to precise the notion of generic uniform observability, we adapt hereafter the definition of the uniform observability $[9,24]$ to the SBLS:

Definition 1 Structured bilinear system $\left(\Sigma_{\Lambda}\right)$ is generically uniformly observable if, for almost all its realizations and for any input value, any pair of initial states $x^{0}(0)$ and $x^{1}(0)$ are distinguishable by observation of the corresponding outputs $y^{0}(t)$ and $y^{1}(t)$ for $t \geq 0$.

From the latter definition, we deduce that a structured system is generically uniformly observable iff for almost all its realizations and for all the input values we can express $x(t)$ in function of $y(t)$, 
$u(t)$ and their derivatives i.e. there always exists a nonsingular function $f$ such that:

$$
x(t)=f\left(y(t), \dot{y}(t), y^{(2)}(t), \ldots, y^{\left(\kappa_{y}\right)}(t), u(t), \dot{u}(t), u^{(2)}(t), \ldots, u^{\left(\kappa_{u}\right)}(t)\right)
$$

Indeed, according to Definition 1, if a structured system is generically uniformly observable then we can always (for all the input values) express its initial conditions in function of the output and its derivatives. In this case, using the system's dynamics $\left(\Sigma_{\Lambda}\right)$, we can write an expression of the form (2). Reciprocally, if we can write an expression like (2), then we can determine in a unique way, using the system's dynamics $\left(\Sigma_{\Lambda}\right)$, the initial conditions $x(0)$ in function of the corresponding output $y(t)$, whatever the value of the known input $u(t)$.

In the case of bilinear systems, since $y(t)$ is a linear function of $x(t)$ and since the dynamics are jointly linear in the state and the input, if we have an expression of $x(t)$ in function of the measured variables, then it is necessarily in the form:

$$
x(t)=\Gamma\left(u(t), \dot{u}(t), u^{(2)}(t), \ldots, u^{\left(\kappa_{u}\right)}(t)\right)\left(\begin{array}{c}
y(t) \\
\dot{y}(t) \\
\vdots \\
y^{\left(K_{y}\right)}(t)
\end{array}\right)
$$

$\Gamma\left(u(t), \dot{u}(t), u^{(2)}(t), \ldots, u^{\left(\kappa_{u}\right)}(t)\right)$ is a matrix whose elements are polynomials in the input components and their derivatives, $\kappa_{y}<n$ and $\kappa_{u}<n$.

From the previous settings, a system is uniformly observable if and only if we can deduce from the equations $\left(\Sigma_{\Lambda}\right)$, according to the differentiation of output $y(t)$, a nonsingular system parameterized by the input and its derivatives and where the unknown variables are the state components.

Hereafter, we enounce a first proposition which allows to define a triangular form ensuring the generic uniform observability of a bilinear system:

Proposition 1 A structured bilinear system is generically uniformly observable if there exist matrices $\Gamma_{0}^{\lambda}, \Gamma_{1}^{\lambda}, \ldots, \Gamma_{K}^{\lambda}$ such that we can generically deduce from equations $\left(\Sigma_{\Lambda}\right)$ relations of the form:

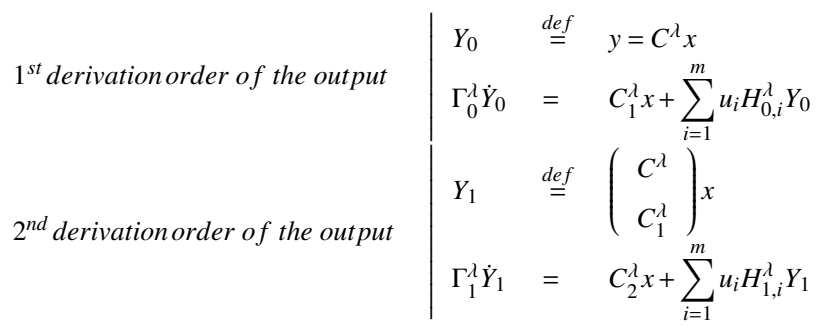

$$
\begin{aligned}
& k^{\text {th }} \text { derivationorder of the output } \mid \begin{array}{c}
Y_{k-1} \stackrel{\text { def }}{=}\left(\begin{array}{c}
C^{\lambda} \\
C_{k-1}^{\lambda}
\end{array}\right) x \\
\Gamma_{k-1}^{\lambda} \dot{Y}_{k-1}=
\end{array} \\
& Y_{k} \stackrel{\text { def }}{=}\left(\begin{array}{l}
C^{\lambda} \\
C_{k}^{\lambda}
\end{array}\right) x
\end{aligned}
$$


with g_rank $\left(\begin{array}{c}C^{\lambda} \\ C_{1}^{\lambda} \\ \vdots \\ C_{k}^{\lambda}\end{array}\right)=$ n.Note that, by construction of form (4), g_rank $\left(\begin{array}{c}C^{\lambda} \\ C_{1}^{\lambda} \\ \vdots \\ C_{k}^{\lambda}\end{array}\right)=g \_$rank $\left(\begin{array}{c}C^{\lambda} \\ C_{k}^{\lambda}\end{array}\right)$.

Here g_rank $\left(M^{\lambda}\right)$ denotes the generic rank $[8,12,17,18]$ of structured matrix $M^{\lambda}$. Roughly speaking the generic rank of a structured matrix $M^{\lambda}$ with $h$ free parameters $\lambda_{1}, \ldots, \lambda_{h}$ is defined as $\max _{\left(\lambda_{1}, \ldots, \lambda_{h}\right)^{T} \in \mathbb{R}^{h}} \operatorname{rank}\left(M^{\lambda}\right)$.

Inform (4), to be coherent with $\left(\Sigma_{\Lambda}\right)$ 's equations, $C_{1}^{\lambda} \stackrel{\text { def }}{=} \Gamma_{0}^{\lambda} C^{\lambda} A^{\lambda}, H_{0, i}^{\lambda}$ is such that $H_{0, i}^{\lambda} C^{\lambda}=\Gamma_{0}^{\lambda} C^{\lambda} B_{i}^{\lambda}$ and for $j=1, \ldots, k-1, C_{j+1}^{\lambda} \stackrel{\text { def }}{=} \Gamma_{j}^{\lambda}\left(\begin{array}{c}C^{\lambda} \\ C_{j}^{\lambda}\end{array}\right) A^{\lambda}$ and $H_{j+1, i}^{\lambda}$ is such that $H_{j+1, i}^{\lambda}\left(\begin{array}{c}C^{\lambda} \\ C_{j}^{\lambda}\end{array}\right)=\Gamma_{j}^{\lambda}\left(\begin{array}{c}C^{\lambda} \\ C_{j}^{\lambda}\end{array}\right) B_{i}^{\lambda}$.

Proof:

Assume that we can put the system into the form (4), then we can write

$$
\left(\begin{array}{c}
C^{\lambda} \\
C_{1}^{\lambda} \\
C_{2}^{\lambda} \\
\vdots \\
C_{k}^{\lambda}
\end{array}\right) x(t)=\left(\begin{array}{c}
y(t) \\
\Gamma_{0}^{\lambda} \dot{y}(t) \\
\Gamma_{1}^{\lambda} \dot{Y}_{1}(t) \\
\vdots \\
\Gamma_{k-1}^{\lambda} \dot{Y}_{k-1}(t)
\end{array}\right)-\left(\begin{array}{c}
0 \\
\sum_{i=1}^{m} u_{i}(t) H_{0, i}^{\lambda} Y_{0}(t) \\
\sum_{i=1}^{m} u_{i}(t) H_{1, i}^{\lambda} Y_{1}(t) \\
\vdots \\
\sum_{i=1}^{m} u_{i}(t) H_{k-1, i}^{\lambda} Y_{k-1}(t)
\end{array}\right)
$$

Since we have $Y_{0}(t)=y(t)$ and for $j \geq 1, Y_{j}(t)=\left(\begin{array}{l}y(t) \\ \Gamma_{j-1}^{\lambda} \dot{Y}_{j-1}(t)-\sum_{i=1}^{m} u_{i}(t) H_{j-1, i}^{\lambda} Y_{j-1}(t)\end{array}\right)$, then all the $Y_{j}$ can be expressed in function of the output, the input and their derivatives i.e. $Y_{j}(t)=\phi_{j}\left(u(t), \dot{u}(t), \ldots, u^{(j)}(t)\right)\left(\begin{array}{c}y(t) \\ \dot{y}(t) \\ \vdots \\ y^{(j)}(t)\end{array}\right)$.

Therefore, we can write $\left(\begin{array}{c}C^{\lambda} \\ C_{1}^{\lambda} \\ \vdots \\ C_{k}^{\lambda}\end{array}\right) x(t)=\Phi\left(u(t), \dot{u}, u^{(2)}(t), \ldots, u^{\left(K_{u}\right)}(t)\right)\left(\begin{array}{c}y(t) \\ \dot{y}(t) \\ \vdots \\ y^{\left(K_{y}\right)}(t)\end{array}\right)$ as the generic rank of $\Gamma_{x} \stackrel{\text { def }}{=}\left(\begin{array}{c}C^{\lambda} \\ C_{1}^{\lambda} \\ \vdots \\ C_{k}^{\lambda}\end{array}\right)$ is equal to $n=\operatorname{dim}(x)$, we can write $x(t)=\left(\Gamma_{x}^{T} \Gamma_{x}\right)^{-1} \Phi\left(u(t), \dot{u}, u^{(2)}(t), \ldots, u^{\left(\kappa_{u}\right)}(t)\right)\left(\begin{array}{c}y(t) \\ \dot{y}(t) \\ \vdots \\ y^{\left(\kappa_{y}\right)}(t)\end{array}\right)$. Consequently, starting from equations (4) we obtain, for any input value, an expression on the form (3). This concludes to the generic uniform observability of structured system $\left(\Sigma_{\Lambda}\right)$.

Remark 1 For single output systems as it was proved in [9], the condition of Proposition 1 is necessary and sufficient to ensure the generic observability of a bilinear system. Nevertheless, in the case of multi-output systems, the following counter-example shows that this condition is not 
necessary:

$$
\begin{aligned}
\dot{x}_{1} & =\lambda_{1} x_{3}+\lambda_{2} u_{1} x_{3}+\lambda_{3} x_{4} \\
\dot{x}_{2} & =\lambda_{4} x_{3}+\lambda_{5} u_{1} x_{4} \\
\dot{x}_{3} & =\lambda_{7} x_{4} \\
\dot{x}_{4} & =\lambda_{8} x_{3} \\
y_{1} & =\lambda_{9} x_{1} \\
y_{2} & =\lambda_{10} x_{2}
\end{aligned}
$$

This system cannot be put on the form 4 of the paper. The observability matrix (by differentiating twice each output) is equal to

$$
O(u, \dot{u})=\left(\begin{array}{cccc}
\lambda_{9} & 0 & 0 & 0 \\
0 & \lambda_{10} & 0 & 0 \\
0 & 0 & \lambda_{9}\left(\lambda_{1}+\lambda_{2} u_{1}\right) & \lambda_{9} \lambda_{3} \\
0 & 0 & \lambda_{10} \lambda_{4} & \lambda_{10} \lambda_{5} u_{1} \\
0 & 0 & \lambda_{9}\left(\lambda_{2} \dot{u}_{1}+\lambda_{3} \lambda_{8}\right) & \lambda_{9} \lambda_{7}\left(\lambda_{1}+\lambda_{2} u_{1}\right) \\
0 & 0 & \lambda_{10} \lambda_{5} \lambda_{8} u_{1} & \lambda_{10}\left(\lambda_{4} \lambda_{7}+\lambda_{5} \dot{u}_{1}\right)
\end{array}\right)
$$

To establish the non-uniform observability of system (6) we must have simultaneously: $\operatorname{det}\left(\begin{array}{cc}\lambda_{1}+\lambda_{2} u_{1} & \lambda_{3} \\ \lambda_{4} & \lambda_{5} u_{1}\end{array}\right)=0, \operatorname{det}\left(\begin{array}{cc}\lambda_{1}+\lambda_{2} u_{1} & \lambda_{3} \\ \lambda_{2} \dot{u}_{1}+\lambda_{3} \lambda_{8} & \lambda_{7}\left(\lambda_{1}+\lambda_{2} u_{1}\right)\end{array}\right)=0, \operatorname{det}\left(\begin{array}{cc}\lambda_{1}+\lambda_{2} u_{1} & \lambda_{3} \\ \lambda_{5} \lambda_{8} u_{1} & \lambda_{4} \lambda_{7}+\lambda_{5} \dot{u}_{1}\end{array}\right)=$ $0, \quad \operatorname{det}\left(\begin{array}{cc}\lambda_{4} & \lambda_{5} u_{1} \\ \lambda_{2} \dot{u}_{1}+\lambda_{3} \lambda_{8} & \lambda_{7}\left(\lambda_{1}+\lambda_{2} u_{1}\right)\end{array}\right)=0, \quad \operatorname{det}\left(\begin{array}{cc}\lambda_{4} & \lambda_{5} u_{1} \\ \lambda_{5} \lambda_{8} u_{1} & \lambda_{4} \lambda_{7}+\lambda_{5} \dot{u}_{1}\end{array}\right)=0 \quad$ and $\operatorname{det}\left(\begin{array}{cc}\lambda_{2} \dot{u}_{1}+\lambda_{3} \lambda_{8} & \lambda_{7}\left(\lambda_{1}+\lambda_{2} u_{1}\right) \\ \lambda_{5} \lambda_{8} u_{1} & \lambda_{4} \lambda_{7}+\lambda_{5} \dot{u}_{1}\end{array}\right)=0$.

Generically, $u$ and $\dot{u}$ cannot verify simultaneously all these equations. So, System (6) is generically uniformly observable.

In the present work, we are interested in the question whether or not $\operatorname{SBLS}\left(\Sigma_{\Lambda}\right)$ can be generically transformed into the form (4) using a graph-theoretic approach. It turns out that this question can be answered by checking some simple graphic conditions. These conditions are sufficient to the generic uniform observability of SBLS $\left(\Sigma_{\Lambda}\right)$. Note that we provide also a necessary condition to the generic uniform observability of $\operatorname{SBLS}\left(\Sigma_{\Lambda}\right)$.

Before presenting the main results of the paper, we give, in the next section, some definitions related to a graphical representation of SBLS. 


\section{Graphical representation of a structured bilinear system}

This section is devoted to the definition of the digraph which is used to represent structured system $\left(\Sigma_{\Lambda}\right)$. This digraph is noted $\mathcal{G}\left(\Sigma_{\Lambda}\right)$ and is constituted by a vertex set $\mathcal{V}$ and an edge set $\mathcal{E}$. More precisely, the vertex set is defined by: $\mathcal{V}=\mathbf{X} \cup \mathbf{X U} \cup \mathbf{Y} \cup \mathbf{Z}$, where $\mathbf{X}=\left\{\mathbf{x}_{\mathbf{1}}, \ldots, \mathbf{x}_{\mathbf{n}}\right\}$ is the set of state vertices, $\mathbf{X U}=\left\{\mathbf{x}_{\mathbf{j}} \mathbf{u}_{\mathbf{i}} \mid \exists k \in\{1, \ldots, n\}\right.$ such that $\left.B_{i}^{\lambda}(k, j) \neq 0\right\}$ is the set of $X$-U mixed vertices, $\mathbf{Y}=\left\{\mathbf{y}_{\mathbf{1}}, \ldots, \mathbf{y}_{\mathbf{p}}\right\}$ is the set of output vertices, $\mathbf{Z}=\left\{\mathbf{z}_{\mathbf{1}}, \ldots, \mathbf{z}_{\mathbf{n}}\right\}$ is the set of dynamic variable vertices. The edge set is $\mathcal{E}=I$-edges $\cup A$-edges $\cup C$-edges $\cup B_{i}$-edges with $I$-edges $=\left\{\left(\mathbf{x}_{\mathbf{i}}, \mathbf{z}_{\mathbf{i}}\right), i=1, \ldots, n\right\}$, $A$-edges $=\left\{\left(\mathbf{z}_{\mathbf{i}}, \mathbf{x}_{\mathbf{j}}\right) \mid A^{\lambda}(i, j) \neq 0\right\}, C$-edges $=\left\{\left(\mathbf{y}_{\mathbf{i}}, \mathbf{x}_{\mathbf{j}}\right) \mid C^{\lambda}(i, j) \neq 0\right\}$, for $i=1, \ldots, m, B_{i}$-edges $=$ $\left\{\left(\mathbf{x}_{\mathbf{j}} \mathbf{u}_{\mathbf{i}}, \mathbf{z}_{\mathbf{k}}\right) \mid B_{i}^{\lambda}(k, j) \neq 0\right\}$.

Here $M^{\lambda}(i, j)$ is the $(i, j)$ th element of matrix $M^{\lambda}$ and $\left(\mathbf{s}_{\mathbf{1}}, \mathbf{s}_{2}\right)$ denotes a directed edge from vertex $\mathbf{s}_{\mathbf{1}} \in \mathcal{V}$ to vertex $\mathbf{s}_{\mathbf{2}} \in \mathcal{V}$. Note that to differentiate the state, the output and the dynamic variables from their corresponding vertices, the latter are written in bold fonts.

Hereafter, we illustrate our proposed digraph representation for SBLS with a simple example:

Example 1 Consider the SBLS defined by:

$A^{\lambda}=\left(\begin{array}{ccc}\lambda_{1} & \lambda_{2} & 0 \\ 0 & 0 & \lambda_{3} \\ 0 & \lambda_{4} & \lambda_{5}\end{array}\right), B_{1}^{\lambda}=\left(\begin{array}{ccc}0 & \lambda_{6} & 0 \\ 0 & 0 & 0 \\ 0 & 0 & 0\end{array}\right), B_{2}^{\lambda}=\left(\begin{array}{ccc}0 & \lambda_{7} & 0 \\ 0 & 0 & \lambda_{8} \\ 0 & 0 & 0\end{array}\right)$ and $C^{\lambda}=\left(\begin{array}{ccc}\lambda_{9} & 0 & 0\end{array}\right)$. To such a model, we associate the digraph in figure 1.

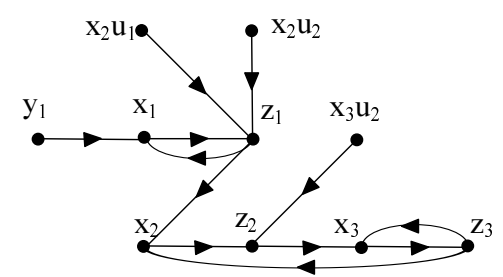

Figure 1: Digraph associated to Example 1

We give now some useful definitions and notations:

- For each edge $e=\left(\mathbf{s}_{\mathbf{0}}, \mathbf{s}_{\mathbf{1}}\right) \in \mathcal{E}, \mathbf{s}_{\mathbf{0}}$ is the begin vertex and $\mathbf{s}_{\mathbf{1}}$ the end vertex of $e$.

- Two edges $e_{1}=\left(\mathbf{s}_{\mathbf{1}}, \mathbf{s}_{\mathbf{1}}^{\prime}\right)$ and $e_{2}=\left(\mathbf{s}_{\mathbf{2}}, \mathbf{s}_{\mathbf{2}}^{\prime}\right)$ elements of $\mathcal{E}$ are $v$-disjoint if $\mathbf{s}_{\mathbf{1}} \neq \mathbf{s}_{\mathbf{2}}$ and $\mathbf{s}_{\mathbf{1}}^{\prime} \neq \mathbf{s}_{\mathbf{2}}^{\prime}$.

Note that $e_{1}$ and $e_{2}$ can be $v$-disjoint even if $\mathbf{s}_{\mathbf{1}}^{\prime}=\mathbf{s}_{\mathbf{2}}$ or $\mathbf{s}_{\mathbf{1}}=\mathbf{s}_{\mathbf{2}}^{\prime}$. In Example $1,\left(\mathbf{x}_{\mathbf{1}}, \mathbf{z}_{\mathbf{1}}\right)$ and $\left(\mathbf{x}_{\mathbf{2}}, \mathbf{z}_{\mathbf{2}}\right)$ are $v$-disjoint. It is also the case for $\left(\mathbf{y}_{1}, \mathbf{x}_{1}\right)$ and $\left(\mathbf{x}_{1}, \mathbf{z}_{1}\right)$. Nevertheless, neither $\left(\mathbf{z}_{1}, \mathbf{x}_{1}\right)$ and $\left(\mathbf{z}_{1}, \mathbf{x}_{2}\right)$ nor $\left(\mathbf{x}_{2} \mathbf{u}_{1}, \mathbf{z}_{1}\right)$ and $\left(\mathbf{x}_{2} \mathbf{u}_{2}, \mathbf{z}_{1}\right)$ are $v$-disjoint.

- Some edges are $v$-disjoint if they are mutually $v$-disjoint.

- A subgraph $\mathcal{S}_{\mathcal{G}}$ of $\mathcal{G}\left(\Sigma_{\Lambda}\right)$ is defined by an edge subset $\mathcal{S}_{\mathcal{E}} \subseteq \mathcal{E}$ and a vertex subset $\mathcal{S}_{\mathcal{V}} \subseteq \mathcal{V}$ such 
that $\mathcal{S}_{\mathcal{V}}$ is constituted by the begin and the end vertices of all the elements of $\mathcal{S}_{\mathcal{E}}$.

- An edge $e$ belongs to a subgraph $\mathcal{S}_{\mathcal{G}}=\left(\mathcal{S}_{\mathcal{E}}, \mathcal{S}_{\mathcal{V}}\right)$ if $e \in \mathcal{S}_{\mathcal{E}}$. A subgraph $\mathcal{S}_{\mathcal{G}}$ of $\mathcal{G}\left(\Sigma_{\Lambda}\right)$ is said to cover a vertex $\mathbf{s}$ if there exists an edge $e$ belonging to $\mathcal{S}_{\mathcal{G}}$ such that $\mathbf{s}$ is the end vertex of $e$.

- A subgraph $\mathcal{S}_{\mathcal{G}}$ of $\mathcal{G}\left(\Sigma_{\Lambda}\right)$ is a $v$-disjoint subgraph if all its edges are $v$-disjoint.

- For any $\mathcal{V}_{0} \subseteq \mathcal{V}$, we denote by $2^{\mathcal{V}_{0}}$ the power set of $\mathcal{V}_{0}$ composed of all the subsets of $\mathcal{V}_{0}$. Function $\varphi$ is defined by:

$$
\begin{aligned}
\varphi: \quad 2^{\mathcal{V}} & \rightarrow 2^{\mathcal{V}} \\
\mathbf{V}_{\mathbf{0}} & \mapsto \mathbf{V}_{\mathbf{1}}=\varphi\left(\mathbf{V}_{\mathbf{0}}\right)=\left\{\mathbf{s} \in \mathcal{V} / \exists \mathbf{s}_{\mathbf{0}} \in \mathbf{V}_{\mathbf{0}} \text { where }\left(\mathbf{s}_{\mathbf{0}}, \mathbf{s}\right) \in \mathcal{E}\right\}
\end{aligned}
$$

Roughly speaking, $\varphi\left(\mathbf{V}_{\mathbf{0}}\right)$ represents the reachable set from $\mathbf{V}_{\mathbf{0}}$ using any edge of $\mathcal{E}$ beginning at $V_{0}$. In Example 1, $\varphi\left(\left\{\mathbf{y}_{\mathbf{1}}, \mathbf{z}_{\mathbf{3}}\right\}\right)=\left\{\mathbf{x}_{\mathbf{1}}, \mathbf{x}_{\mathbf{2}}, \mathbf{x}_{\mathbf{3}}\right\}$. We define also function $\bar{\varphi}$ such that:

$$
\begin{aligned}
\bar{\varphi}: & 2^{\mathbf{Z}} \rightarrow 2^{\mathbf{X} \cup \mathbf{X U}} \\
\mathbf{V}_{\mathbf{1}} & \mapsto \overline{\mathbf{V}}_{\mathbf{0}}=\bar{\varphi}\left(\mathbf{V}_{\mathbf{1}}\right)=\left\{\mathbf{s} \in \mathbf{X} \cup \mathbf{X U} / \exists \mathbf{s}_{\mathbf{1}} \in \mathbf{V}_{\mathbf{1}} \text { where }\left(\mathbf{s}, \mathbf{s}_{\mathbf{1}}\right) \in \mathcal{E}\right\}
\end{aligned}
$$

In Example 1, $\bar{\varphi}\left(\left\{\mathbf{z}_{2}, \mathbf{z}_{3}\right\}\right)=\left\{\mathbf{x}_{2}, \mathbf{x}_{3}, \mathbf{x}_{3} \mathbf{u}_{2}\right\}$.

- $\alpha$ is the function defined by:

$$
\begin{aligned}
\alpha: 2^{\mathbf{X U}} & \rightarrow 2^{\mathbf{X}} \\
\mathbf{V}_{\mathbf{X U}} & \mapsto \mathbf{V}_{\mathbf{X}}=\alpha\left(\mathbf{V}_{\mathbf{X U}}\right)=\left\{\mathbf{x}_{\mathbf{i}} \in \mathbf{X} / \exists j \in\{1, \ldots, m\} \text { such that } \mathbf{x}_{\mathbf{i}} \mathbf{u}_{\mathbf{j}} \in \mathbf{V}_{\mathbf{X U}}\right\}
\end{aligned}
$$

In Example 1, $\alpha\left(\left\{\mathbf{x}_{2} \mathbf{u}_{1}, \mathbf{x}_{2} \mathbf{u}_{2}\right\}\right)=\left\{\mathbf{x}_{\mathbf{2}}\right\}$.

\section{Main results}

In order to graphically analyse the generic uniform observability of SBLS, we follow an iterative approach where the $i^{\text {th }}$ stage corresponds to the $i^{\text {th }}$ differentiation order of the output.

Hereafter, we detail the first step of our approach. As it is shown in the form (4), only combinations $\Gamma_{0}^{\lambda} y$ such that we can deduce from $\left(\Sigma_{\Lambda}\right)$ an equation of the form:

$$
\Gamma_{0}^{\lambda} \dot{y}=C_{1}^{\lambda} x+\sum_{i=1}^{m} u_{i} H_{0, i}^{\lambda} Y_{0}, \quad Y_{0} \stackrel{\text { def }}{=} y=C^{\lambda} x
$$

are useful. In equation (10), $C_{1}^{\lambda}=\Gamma_{0}^{\lambda} C^{\lambda} A^{\lambda}$ and $H_{0, i}^{\lambda} C^{\lambda}=\Gamma_{0}^{\lambda} C^{\lambda} B_{i}^{\lambda}$. Determining these combinations amounts to find the combined vertices associated to some combinations $P^{\lambda} z$ which satisfy $P^{\lambda} z=\Gamma_{0}^{\lambda} \dot{y}-\sum_{i=1}^{m} u_{i} H_{0, i}^{\lambda} y$. These vertices are called useful vertices. The first part of this section is dedicated to the determination of these useful vertices.

Let us first precise the notion of combined vertices corresponding to combinations $P^{\lambda} z$ :

- We denote by $\mathbf{z}_{\mathbf{i}_{1}, \mathbf{i}_{2}, \ldots, \mathbf{i}_{\mathbf{k}}}$ the vertex associated to any combination of dynamic variables 
$z_{i_{1}}, z_{i_{2}}, \ldots, z_{i_{k}}$ whatever $i_{1}<i_{2}<\ldots<i_{k} . \mathbf{z}_{\mathbf{i}_{1}, \mathbf{i}_{2}, \ldots, \mathbf{i}_{\mathbf{k}}}$, which we call combined vertex, can be defined by means of bijective function $\pi$ associating a combined vertex to each nonempty subset of $\mathbf{Z}:\left\{\mathbf{z}_{\mathbf{i}_{1}}, \mathbf{z}_{\mathbf{i}_{2}}, \ldots, \mathbf{z}_{\mathbf{i}_{\mathbf{k}}}\right\} \mapsto \pi\left(\left\{\mathbf{z}_{\mathbf{i}_{1}}, \mathbf{z}_{\mathbf{i}_{2}}, \ldots, \mathbf{z}_{\mathbf{i}_{\mathbf{k}}}\right\}\right) \stackrel{\text { def }}{=} \mathbf{z}_{\mathbf{i}_{1}, \mathbf{i}_{\mathbf{2}}, \ldots, \mathbf{i}_{\mathbf{k}}}$. We denote by $\bar{\pi}$ the inverse function of $\pi$. The set of combined vertices is noted $\mathbf{Z}_{\mathbf{C}}$. Obviously, $\mathbf{Z} \subseteq \mathbf{Z}_{\mathbf{C}}$.

- For each vertex subset $\mathbf{V}_{\mathbf{0}} \subseteq \mathcal{V} \cup \mathbf{Z}_{\mathbf{C}}$, $\operatorname{card}\left(\mathbf{V}_{\mathbf{0}}\right)$ represents the cardinality of $\mathbf{V}_{\mathbf{0}}$ i.e. the number of vertices included in $\mathbf{V}_{\mathbf{0}}$.

- A nonempty vertex subset $\mathbf{V}_{\mathbf{C}} \subseteq \mathbf{Z}_{\mathbf{C}}$ is said "structurally minimal" if

$$
\forall \overline{\mathbf{V}} \subseteq \mathbf{V}_{\mathbf{C}}, \quad \operatorname{card}\left(\bigcup_{\mathbf{v} \in \overline{\mathbf{V}}} \bar{\pi}(\mathbf{v})\right)-\operatorname{card}(\overline{\mathbf{V}}) \geq \max _{\mathbf{v} \in \overline{\mathbf{V}}}[\operatorname{card}(\bar{\pi}(\mathbf{v}))]-1
$$

For example, $\mathbf{V}_{1}=\left\{\mathbf{z}_{\mathbf{1}}, \mathbf{z}_{2,3}, \mathbf{z}_{2,4}\right\}$ is structurally minimal while $\mathbf{V}_{2}=\left\{\mathbf{z}_{1,2,3}, \mathbf{z}_{2,3}, \mathbf{z}_{2,4}\right\}$ is not structurally minimal because for $\overline{\mathbf{V}}=\left\{\mathbf{z}_{\mathbf{1}, 2,3}, \mathbf{z}_{2,3}\right\}$, card $\left(\bigcup_{\mathbf{v} \in \overline{\mathbf{V}}} \bar{\pi}(\mathbf{v})\right)-\operatorname{card}(\overline{\mathbf{V}})=3-2$ and $\max _{\mathbf{v} \in \overline{\mathbf{V}}}(\operatorname{card}(\bar{\pi}(\mathbf{v})))-1=3-1$. Similarly, $\mathbf{V}_{3}=\left\{\mathbf{z}_{1,2}, \mathbf{z}_{1,3}, \mathbf{z}_{2,3}\right\}$ is not structurally minimal because for $\overline{\mathbf{V}}=\mathbf{V}_{3}$, condition (11) is not satisfied.

The notion of structural minimality is related to the notion of vector linear independence. Indeed, consider a structurally minimal vertex subset $\mathbf{V}_{\mathbf{Z}}=\left\{\mathbf{z}_{\Omega_{1}}, \mathbf{z}_{\Omega_{2}}, \ldots, \mathbf{z}_{\Omega_{\mathbf{k}}}\right\} \subseteq \mathbf{Z}_{C}$ and its associated vector $\tilde{z}=\left(z_{\Omega_{1}}, z_{\Omega_{2}}, \ldots, z_{\Omega_{k}}\right)^{T}=\left(P_{1}^{\lambda} z, P_{2}^{\lambda} z, \ldots, P_{k}^{\lambda} z\right)^{T}$, where $P_{i}^{\lambda} \in \mathbf{R}^{1 \times n}$. We can easily prove that the family of vectors $\left(P_{1}^{\lambda}\right)^{T}, \ldots,\left(P_{k}^{\lambda}\right)^{T}$ is generically linearly independent because of the structural minimality of $\mathbf{V}_{\mathbf{Z}}$.

- To each $\mathbf{V}_{\mathbf{C}} \subseteq \mathbf{Z}_{\mathbf{C}}$, we can associate a vertex subset $\wp\left(\mathbf{V}_{\mathbf{C}}\right)$ constituted by all the elements $\mathbf{s} \in \mathbf{Z}_{\mathbf{C}}$ for which there exists $\mathbf{V}_{\mathbf{0}} \subseteq \mathbf{V}_{\mathbf{C}}$ such that:

$$
\left\{\begin{array}{l}
\operatorname{card}\left(\bigcup_{\mathbf{v} \in \mathbf{V}_{\mathbf{0}}} \bar{\pi}(\mathbf{v})\right)-\operatorname{card}(\bar{\pi}(\mathbf{s}))=\operatorname{card}\left(\mathbf{V}_{\mathbf{0}}\right)-1, \\
\forall \overline{\mathbf{V}}_{\mathbf{0}} \subset \mathbf{V}_{\mathbf{0}} \mid \operatorname{card}\left(\overline{\mathbf{V}}_{\mathbf{0}}\right)=\operatorname{card}\left(\mathbf{V}_{\mathbf{0}}\right)-1, \bigcup_{\mathbf{v} \in \overline{\mathbf{V}}_{\mathbf{0}}} \bar{\pi}(\mathbf{v}) \cup \bar{\pi}(\mathbf{s})=\bigcup_{\mathbf{v} \in \mathbf{V}_{\mathbf{0}}} \bar{\pi}(\mathbf{v})
\end{array}\right.
$$

For example, for $\mathbf{V}_{\mathbf{C}}=\left\{\mathbf{z}_{\mathbf{1}, \mathbf{2}}, \mathbf{z}_{\mathbf{1}, \mathbf{3}}, \mathbf{z}_{\mathbf{4}}\right\}$, we have $\wp\left(\mathbf{V}_{\mathbf{C}}\right)=\left\{\mathbf{z}_{\mathbf{1}, \mathbf{2}}, \mathbf{z}_{\mathbf{1}, \mathbf{3}}, \mathbf{z}_{\mathbf{2}, \mathbf{3}}, \mathbf{z}_{\mathbf{4}}\right\}$. Note that $\forall \mathbf{V}_{\mathbf{Z}} \subseteq \mathbf{Z}$ we have $\wp\left(\mathbf{V}_{\mathbf{Z}}\right)=\mathbf{V}_{\mathbf{Z}}$. Obviously, we have $\forall \mathbf{V}_{\mathbf{C}} \subseteq \mathbf{Z}_{\mathbf{C}}, \mathbf{V}_{\mathbf{C}} \subseteq \wp\left(\mathbf{V}_{\mathbf{C}}\right)$.

- The definition of $\varphi$ in section 3 is adapted for taking into account $\mathbf{Z}_{\mathbf{C}}$. In the sequel, $\varphi_{e}$ is such that:

$$
\begin{aligned}
\varphi_{e}: 2^{\mathcal{V} \cup \mathbf{Z}_{\mathbf{C}}} & \rightarrow 2^{\mathcal{V}} \\
\mathbf{V}_{\mathbf{0}} & \mapsto \mathbf{V}_{\mathbf{1}}=\varphi_{e}\left(\mathbf{V}_{\mathbf{0}}\right)=\left(\bigcup_{s \in \mathbf{V}_{\mathbf{0}} \cap \mathbf{Z}_{\mathbf{C}}} \varphi(\bar{\pi}(s))\right) \cup \varphi\left(V_{0} \cap \mathcal{V}\right)
\end{aligned}
$$


In Example 1, $\varphi_{e}\left(\left\{\mathbf{y}_{\mathbf{1}}, \mathbf{z}_{\mathbf{2}, \mathbf{3}}\right\}\right)=\varphi\left(\left\{\mathbf{y}_{\mathbf{1}}\right\}\right) \cup \varphi\left(\left\{\mathbf{z}_{\mathbf{2}}\right\}\right) \cup \varphi\left(\left\{\mathbf{z}_{\mathbf{3}}\right\}\right)=\left\{\mathbf{x}_{\mathbf{1}}, \mathbf{x}_{\mathbf{2}}, \mathbf{x}_{\mathbf{3}}\right\}$

We define, finally, function $\theta$ as:

$$
\begin{aligned}
\theta: \quad 2^{\mathbf{Y} \cup \mathbf{Z}_{\mathbf{C}}} & \rightarrow \mathbb{N} \\
& \mathbf{V}_{\mathbf{0}}
\end{aligned}
$$

knowing that, to take into account $\mathbf{Z}_{\mathbf{C}}$, we add virtually (there is no need to redraw the digraph associated to the system) to the digraph the following edge subset $A_{C}$-edges $=$

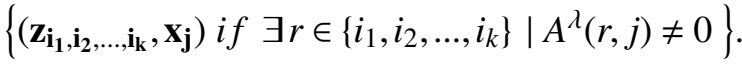

In Example $1, \theta(\mathbf{Y} \cup \mathbf{Z})=3\left(\left(\mathbf{y}_{1}, \mathbf{x}_{1}\right),\left(\mathbf{z}_{1}, \mathbf{x}_{2}\right),\left(\mathbf{z}_{2}, \mathbf{x}_{3}\right)\right.$ are $3 v$-disjoint edges starting from $\left.\mathbf{Y} \cup \mathbf{Z}\right)$ and $\theta\left(\left\{\mathbf{z}_{1}, \mathbf{z}_{2,3}\right\}\right)=2\left(\left(\mathbf{z}_{1}, \mathbf{x}_{\mathbf{2}}\right),\left(\mathbf{z}_{2,3}, \mathbf{x}_{\mathbf{3}}\right)\right.$ are $2 v$-disjoint edges starting from $\left.\left\{\mathbf{z}_{1}, \mathbf{z}_{2,3}\right\}\right)$.

Function $\theta$ has the following property:

P1. Consider $\mathbf{V}_{\mathbf{Y}}=\left\{\mathbf{y}_{\mathbf{i}_{1}}, \mathbf{y}_{\mathbf{i}_{2}}, \ldots, \mathbf{y}_{\mathbf{i}_{\mathbf{k}}}\right\} \subseteq \mathbf{Y}$ and its associated vector $\tilde{y}=\left(y_{i_{1}}, y_{i_{2}}, \ldots, y_{i_{k}}\right)^{T}$. Let us denote by $\tilde{C}^{\lambda}$ the matrix such that $\tilde{y}=\tilde{C}^{\lambda} x$, then from the results on the matrices generic rank $[18,23]$, $\theta\left(\mathbf{V}_{\mathbf{Y}}\right)=g \_\operatorname{rank}\left(\tilde{C}^{\lambda}\right)$.

Similarly, consider a structurally minimal vertex subset $\mathbf{V}_{\mathbf{Z}}=\left\{\mathbf{z}_{\Omega_{1}}, \mathbf{z}_{\Omega_{2}}, \ldots, \mathbf{z}_{\Omega_{\mathbf{k}}}\right\} \subseteq \mathbf{Z}_{C}$ and its associated vector $\tilde{z}=\left(z_{\Omega_{1}}, z_{\Omega_{2}}, \ldots, z_{\Omega_{k}}\right)^{T}=\left(P_{1}^{\lambda} z, P_{2}^{\lambda} z, \ldots, P_{k}^{\lambda} z\right)^{T}$, where $P_{i}^{\lambda} \in \mathbf{R}^{1 \times n}$. Let $\tilde{E}^{\lambda}=\left(\begin{array}{c}P_{1}^{\lambda} \\ P_{2}^{\lambda} \\ \vdots \\ P_{k}^{\lambda}\end{array}\right) A^{\lambda}$. We have then $\tilde{z}=\tilde{E}^{\lambda} x$ and so $\theta\left(\mathbf{V}_{\mathbf{Z}}\right)=\mathrm{g} \_\operatorname{rank}\left(\tilde{E}^{\lambda}\right)$.

This property can be directly deduced from the results of [23] or from the results related to the maximum matching notion [17].

Using function $\theta$, we define a particular vertex set $\mathbf{V}_{\mathbf{M}^{0}}^{*}$ :

Definition 2 Let $\mathbf{V}_{\mathbf{M}^{0}}^{*}$ be a subset of $\mathbf{Y}$ such that

$$
\left\{\begin{array}{l}
\theta\left(\mathbf{V}_{\mathbf{M}^{\mathbf{0}}}^{*}\right)=\operatorname{card}\left(\varphi_{e}\left(\mathbf{V}_{\mathbf{M}^{\mathbf{0}}}^{*}\right)\right) \\
\operatorname{card}\left(\mathbf{V}_{\mathbf{M}^{\mathbf{0}}}^{*}\right) \text { is maximal }
\end{array}\right.
$$

Moreover, we take the following notations $\mathbf{V}_{\mathbf{X}^{\mathbf{0}}}^{*}=\varphi_{e}\left(\mathbf{V}_{\mathbf{M}^{0}}^{*}\right)$ and $\mathbf{V}_{\mathbf{X U}^{\mathbf{0}}}^{*}=$ $\left\{\mathbf{s} \in \mathbf{X U}\right.$ such that,$\left.\alpha(\{\mathbf{s}\}) \subseteq \mathbf{V}_{\mathbf{X}^{\mathbf{0}}}^{*}\right\}$.

If $\mathbf{V}_{\mathbf{M}^{0}}^{*}$ exists, then it is unique because if relation $\theta\left(\mathbf{V}_{\mathbf{M}}\right)=\operatorname{card}\left(\varphi_{e}\left(\mathbf{V}_{\mathbf{M}}\right)\right)$ is satisfied for $\mathbf{V}_{\mathbf{M}}=\mathbf{V}_{\mathbf{M}_{\mathbf{1}}}$ and for $\mathbf{V}_{\mathbf{M}}=\mathbf{V}_{\mathbf{M}_{2}}$, then it is also satisfied for $\mathbf{V}_{\mathbf{M}}=\mathbf{V}_{\mathbf{M}_{1}} \cup \mathbf{V}_{\mathbf{M}_{2}}$.

We give now, in the following lemma, the necessary and sufficient conditions to be satisfied in order to express state component $x_{i}$ by means of output $y$ :

Lemma 1 Consider structured bilinear system $\left(\Sigma_{\Lambda}\right)$ represented by digraph $\mathcal{G}\left(\Sigma_{\Lambda}\right)$. State component $x_{i}$ can be generically expressed in function of the output i.e. there exists a matrix $T_{i}^{\lambda}$ such that 
$x_{i}=T_{i}^{\lambda} y$, iff in $\mathcal{G}\left(\Sigma_{\Lambda}\right)$

$$
\exists \mathbf{V}_{\mathbf{Y}} \subseteq \mathbf{Y} \text { such that } \begin{cases}(i) & \mathbf{x}_{\mathbf{i}} \in \varphi_{e}\left(\mathbf{V}_{\mathbf{Y}}\right) \\ (\text { ii }) & \theta\left(\mathbf{V}_{\mathbf{Y}}\right)=\operatorname{card}\left(\varphi_{e}\left(\mathbf{V}_{\mathbf{Y}}\right)\right)\end{cases}
$$

or equivalently $\mathbf{x}_{\mathbf{i}} \in \mathbf{V}_{\mathbf{X}^{0}}^{*}$

\section{Proof:}

Sufficiency: Assume that (16) is satisfied. Let $x_{\Omega}$ be the part of the state vector associated to vertex set $\varphi_{e}\left(\mathbf{V}_{\mathbf{Y}}\right)$ and let $y_{\Omega}$ be the output vector associated to $\mathbf{V}_{\mathbf{Y}}$. According to the graph construction, there exists necessarily a matrix $C_{\Omega}$ such that $y_{\Omega}=C_{\Omega} x_{\Omega}$. If $\theta\left(\mathbf{V}_{\mathbf{Y}}\right)=\operatorname{card}\left(\varphi_{e}\left(\mathbf{V}_{\mathbf{Y}}\right)\right)$ then using Property $\mathbf{P 1}$ of function $\theta$, the generic rank of $C_{\Omega}$ equals $\operatorname{card}\left(\varphi_{e}\left(\mathbf{V}_{\mathbf{Y}}\right)\right)=\operatorname{dim}\left(x_{\Omega}\right)$. Thus, there exists generically a matrix $T_{\Omega}$ of full row rank such that $x_{\Omega}=T_{\Omega} y_{\Omega}$ where $T_{\Omega}$ is the pseudoinverse of $C_{\Omega}$. Since $\mathbf{x}_{\mathbf{i}} \in \varphi_{e}\left(\mathbf{V}_{\mathbf{Y}}\right)$, we have $x_{i}=T_{i}^{\lambda} y$ where $T_{i}^{\lambda}$ is the row of $T_{\Omega}$ corresponding to the state component $x_{i}$ in the equality $x_{\Omega}=T_{\Omega} y_{\Omega}$.

Necessity: Suppose that there exists a matrix $T_{i}^{\lambda}$ such that $x_{i}=T_{i}^{\lambda} y$, then there exists necessarily a square nonsingular linear system of independent equations linking an output subvector $y_{\Omega}$ to a state subvector $x_{\Omega}$ which includes $x_{i}$ i.e. there exists a square nonsingular matrix $T_{\Omega}$ such that $x_{\Omega}=T_{\Omega} y_{\Omega}$. So, $y_{\Omega}=T_{\Omega}^{-1} x_{\Omega}$. Let $\mathbf{V}_{\mathbf{Y}}$ the vertex subset associated to the output components included in $y_{\Omega}$, and $\mathbf{V}_{\mathbf{x}}$ the vertex subset associated to the state components included in $x_{\Omega}$. On the one hand, as $y_{\Omega}=T_{\Omega}^{-1} x_{\Omega}$, we have that $\mathbf{V}_{\mathbf{x}}=\varphi_{e}\left(\mathbf{V}_{\mathbf{Y}}\right)$ and since state subvector $x_{\Omega}$ includes $x_{i}$, we have that $\mathbf{x}_{\mathbf{i}} \in \mathbf{V}_{\mathbf{x}}$. On the other hand, as $T_{\Omega}$ is of full rank, we have that g_rank $\left(T_{\Omega}\right)=$ g_rank $\left(T_{\Omega}^{-1}\right)=\theta\left(\mathbf{V}_{\mathbf{Y}}\right)=\operatorname{card}\left(\mathbf{V}_{\mathbf{X}}\right)=\operatorname{card}\left(\varphi_{e}\left(\mathbf{V}_{\mathbf{Y}}\right)\right)$.

\subsection{Determination of the useful vertices}

If $\theta(\mathbf{Y})=n$, or equivalently $\mathbf{V}_{\mathbf{X}^{\mathbf{0}}}^{*}=\mathbf{X}$, then $\mathrm{g}_{-} \operatorname{rank}\left(C^{\lambda}\right)=n$ and so the system is generically uniformly observable. Otherwise, the first derivative of the output is necessary to achieve the state observation. Hereafter, we study the conditions ensuring that a combination of dynamical variables $z_{i}$ can be expressed using $y$ and $u$ only i.e. there exists generically matrix $P^{\lambda}$ such that $P^{\lambda} z=\Gamma_{0}^{\lambda} \dot{y}-\sum_{i=1}^{m} u_{i} H_{0, i}^{\lambda} y$. This amounts to find matrix $\Gamma_{0}$ of the form (4). To do so, our first objective is to construct a subset of $\mathbf{Z}_{\mathbf{C}}$ which represents all the output combinations having derivatives independent from $x_{i} u_{j}$ if $x_{i}$ cannot be expressed using only $y$. In this respect, the following lemma aims to provide the conditions which guarantee that a combined vertex is useful.

Lemma 2 Consider system $\left(\Sigma_{\Lambda}\right)$, represented by digraph $\mathcal{G}\left(\Sigma_{\Lambda}\right)$. Let $\mathbf{V}_{\mathbf{0}}=\left\{\mathbf{z}_{\mathbf{i}_{1}}, \mathbf{z}_{\mathbf{i}_{2}}, \ldots, \mathbf{z}_{\mathbf{i}_{\mathbf{k}}}\right\}$ be a subset of $\mathbf{Z}$ and note by $\pi\left(\mathbf{V}_{\mathbf{0}}\right)=\mathbf{Z}_{\mathbf{i}_{1}, \mathbf{i}_{\mathbf{2}}, \ldots, \mathbf{i}_{\mathbf{k}}}$ its corresponding combined vertex. Assume that 
$\nexists \mathbf{V}_{\mathbf{0}}^{\prime} \subset \mathbf{V}_{\mathbf{0}}$ such that $\pi\left(\mathbf{V}_{\mathbf{0}}^{\prime}\right)$ is useful.

Combined vertex $\mathbf{z}_{\mathbf{i}_{1}, \mathbf{i}_{2}, \ldots, \mathbf{i}_{\mathbf{k}}}$ is generically useful and there exists vector $P_{k}^{\lambda} \in \mathbb{R}^{1 \times k}$, such that $P_{k}^{\lambda}\left(z_{i_{1}}, z_{i_{2}}, \ldots, z_{i_{k}}\right)^{T}$ can be expressed in function of $\dot{y}$ and $u_{i} y$ only i.e. $P_{k}^{\lambda}\left(z_{i_{1}}, z_{i_{2}}, \ldots, z_{i_{k}}\right)^{T}=$ $\Gamma_{0}^{\lambda} \dot{y}-\sum_{i=1}^{m} u_{i} H_{0, i}^{\lambda} y$ iff $\exists \mathbf{V}_{\mathbf{Y}} \subseteq \mathbf{Y}$ satisfying:

$$
\theta\left(\mathbf{V}_{\mathbf{Y}}\right)+k+\operatorname{card}\left(\bar{\varphi}\left(\mathbf{V}_{\mathbf{0}}\right) \cap \mathbf{V}_{\mathbf{X U}^{0}}^{*}\right)=\operatorname{card}\left(\varphi_{e}\left(\mathbf{V}_{\mathbf{Y}}\right) \cup \bar{\varphi}\left(\mathbf{V}_{\mathbf{0}}\right)\right)+1
$$

\section{Proof:}

Sufficiency: Assume that $\exists \mathbf{V}_{\mathbf{Y}} \subseteq \mathbf{Y}$ such that (17) is satisfied and let us take the following notations:

- $\overline{\mathbf{V}_{\mathbf{X U}^{\mathbf{0}}}^{*}}$ is the complementary set of $\mathbf{V}_{\mathbf{X U}^{\mathbf{0}}}^{*}$ in $\mathbf{X U}$ i.e. $\overline{\mathbf{V}_{\mathbf{X U}^{\mathbf{0}}}^{*}}=\mathbf{X U} \backslash \mathbf{V}_{\mathbf{X U}^{\mathbf{0}}}^{*}$;

- $x_{\Omega}$ is the vector whose components correspond to the elements of subset $\varphi_{e}\left(\mathbf{V}_{\mathbf{Y}}\right) \cup\left(\bar{\varphi}\left(\mathbf{V}_{\mathbf{0}}\right) \cap \mathbf{X}\right)$.

- $x u_{\Omega}$ is the vector whose components correspond to the elements of vertex set $\bar{\varphi}\left(\mathbf{V}_{\mathbf{0}}\right) \cap \overline{\mathbf{V}_{\mathbf{X U ^ { 0 }}}^{*}}$;

- $z_{\Omega}=\left(z_{i_{1}}, z_{i_{2}}, \ldots, z_{i_{k}}\right)^{T}$ is the vector of dynamic variables associated to $\mathbf{V}_{\mathbf{0}}$;

- $X_{\Omega}=\left(\begin{array}{c}\dot{x}_{\Omega} \\ x u_{\Omega}\end{array}\right)$;

- $y_{\Omega}$ is the vector of the output components associated to $\mathbf{V}_{\mathbf{Y}}$.

By construction of graph $\mathcal{G}\left(\Sigma_{\Lambda}\right)$ representing system $\left(\Sigma_{\Lambda}\right), y_{\Omega}$ is a linear function of the state components associated to $\varphi_{e}\left(\mathbf{V}_{\mathbf{Y}}\right)$ and $z_{\Omega}$ is a linear function of the state components associated to $\bar{\varphi}\left(\mathbf{V}_{\mathbf{0}}\right)$. A part of $\bar{\varphi}\left(\mathbf{V}_{\mathbf{0}}\right)$ can be directly written in function of $y$, particularly the part associated to $\bar{\varphi}\left(\mathbf{V}_{\mathbf{0}}\right) \cap \mathbf{V}_{\mathbf{X \mathbf { U } ^ { 0 }}}^{*}$ as it is proven in Lemma 1. Then, using our notations, there exist structured matrices $C_{\Omega}, E_{\Omega}$ and $F_{i, \Omega}$ for $i=1, \ldots, m$ such that we can write $\dot{y}_{\Omega}=C_{\Omega} X_{\Omega}$ and $z_{\Omega}=E_{\Omega} X_{\Omega}+\sum_{i=1}^{m} u_{i} F_{i, \Omega} y_{\Omega}$. Yet, we have that $\operatorname{dim}\left(\operatorname{Im}\left(E_{\Omega}^{T}\right)\right)=k, X_{\Omega} \in \mathbb{R}^{v}$ where $v=$ $\operatorname{card}\left(\varphi_{e}\left(\mathbf{V}_{\mathbf{Y}}\right) \cup \bar{\varphi}\left(\mathbf{V}_{\mathbf{0}}\right)\right)-\operatorname{card}\left(\bar{\varphi}\left(\mathbf{V}_{\mathbf{0}}\right) \cap \mathbf{V}_{\mathbf{X U}^{0}}^{*}\right)$ and from property $\mathbf{P 1}$ of $\theta$, we have that $\theta\left(\mathbf{V}_{\mathbf{Y}}\right)=$ $\operatorname{dim}\left(\operatorname{Im}\left(C_{\Omega}^{T}\right)\right)$. Therefore, relation (17) implies that generically

$$
\operatorname{dim}\left(\operatorname{Im}\left(C_{\Omega}^{T}\right)\right)+\operatorname{dim}\left(\operatorname{Im}\left(E_{\Omega}^{T}\right)\right)=v+1
$$

Obviously, $C_{\Omega}$ and $E_{\Omega}$ have $v$ columns and $\operatorname{so} \operatorname{Im}\left(C_{\Omega}^{T}\right)$ and $\operatorname{Im}\left(E_{\Omega}^{T}\right)$ are subspaces of $\mathbb{R}^{v}$. From equation (18), we can conclude that $\operatorname{dim}\left(\operatorname{Im}\left(E_{\Omega}^{T}\right) \cap \operatorname{Im}\left(C_{\Omega}^{T}\right)\right) \geq 1$. Consequently, generically there exist matrices $P_{k}^{\lambda}$ and $\Gamma_{0}^{\lambda}$ such that $P_{k}^{\lambda} E_{\Omega}=\Gamma_{0}^{\lambda} C_{\Omega} \Rightarrow P_{k}^{\lambda} z_{\Omega}=\Gamma_{0}^{\lambda} \dot{y}-\sum_{i=1}^{m} u_{i} H_{0, i}^{\lambda} y$. This proves the sufficiency of Lemma 2.

$\operatorname{Remark} 2$ Note that $\theta\left(\mathbf{V}_{\mathbf{Y}}\right)+k \geq \operatorname{card}\left(\varphi_{e}\left(\mathbf{V}_{\mathbf{Y}}\right) \cup \bar{\varphi}\left(\mathbf{V}_{\mathbf{0}}\right)\right)+1-\operatorname{card}\left(\bar{\varphi}\left(\mathbf{V}_{\mathbf{0}}\right) \cap \mathbf{V}_{\mathbf{X U}^{0}}^{*}\right)$ is also sufficient to prove the existence of vectors $P_{k}^{\lambda} \in \mathbb{R}^{1 \times k}$ such that $P_{k}^{\lambda}\left(z_{i_{1}}, z_{i_{2}}, \ldots, z_{i_{k}}\right)^{T}=\Gamma_{0}^{\lambda} \dot{y}-\sum_{i=1}^{m} u_{i} H_{0, i}^{\lambda} y$.

Necessity: Assume that there exists vector $P_{k}^{\lambda} \in \mathbb{R}^{k}$, such that $P_{k}^{\lambda} z_{\Omega}=\Gamma_{0}^{\lambda} \dot{y}-\sum_{i=1}^{m} u_{i} H_{0, i}^{\lambda} y$ then at least one combination of components associated to $\bar{\varphi}\left(\mathbf{V}_{\mathbf{0}}\right)$ can be expressed using $\dot{y}_{\Omega}\left(y_{\Omega}\right.$ is the 
vector of components of $y$ which intervenes in $\Gamma_{0}^{\lambda} \dot{y}$ i.e. $\left.\Gamma_{0}^{\lambda} \dot{y}=\Gamma_{0, \Omega}^{\lambda} \dot{y}_{\Omega}\right)$. Let $\mathbf{V}_{\mathbf{Y}}$ be the vertex subset associated to the components of $y_{\Omega}$.

From the construction of the digraph, $P_{k}^{\lambda} z_{\Omega}$ is a linear function of $\dot{x}_{j}$ where $\mathbf{x}_{\mathbf{j}} \in \bar{\varphi}\left(\mathbf{V}_{\mathbf{0}}\right) \cap \mathbf{X}$ and of $x_{k} u_{l}$ where $\mathbf{x}_{\mathbf{k}} \mathbf{u}_{\mathbf{l}} \in \bar{\varphi}\left(\mathbf{V}_{\mathbf{0}}\right) \cap \mathbf{X U}$. To satisfy $P_{k}^{\lambda} z_{\Omega}=\Gamma_{0}^{\lambda} \dot{y}+\sum_{i=1}^{m} u_{i} H_{0, i}^{\lambda} y$, the variables corresponding to $\bar{\varphi}\left(\mathbf{V}_{\mathbf{0}}\right)$ are necessarily either eliminated in combination $P_{k}^{\lambda} z_{\Omega}$ or can be expressed only in function $y$. On the one hand, a combination of $k$ dynamical variables $z_{i}$ can eliminate at most $k-1$ elements of $\bar{\varphi}\left(\mathbf{V}_{\mathbf{0}}\right)$. On the other hand, the variables associated to the vertices of $\bar{\varphi}\left(\mathbf{V}_{\mathbf{0}}\right) \cap \mathbf{V}_{\mathbf{X} \mathbf{U}^{\mathbf{0}}}^{*}$ can be expressed in function of $y$ and $u$ as they are included in $\mathbf{V}_{\mathbf{X U}^{\mathbf{0}}}^{*}$ (Lemma 1). Let $q=\operatorname{card}\left[\bar{\varphi}\left(\mathbf{V}_{\mathbf{0}}\right) \backslash\left(\bar{\varphi}\left(\mathbf{V}_{\mathbf{0}}\right) \cap \mathbf{V}_{\mathbf{X} \mathbf{U}^{0}}^{*}\right)\right]$. Thus, at least $q-k+1$ variables corresponding to the vertices included in $\bar{\varphi}\left(\mathbf{V}_{\mathbf{0}}\right) \backslash\left(\bar{\varphi}\left(\mathbf{V}_{\mathbf{0}}\right) \cap \mathbf{V}_{\mathbf{X} \mathbf{U}^{0}}^{*}\right)$ must be generically expressed in function of $y_{\Omega}$ to satisfy equation $P_{k}^{\lambda} z_{\Omega}=\Gamma_{0, \Omega}^{\lambda} \dot{y}_{\Omega}+\sum_{i=1}^{m} u_{i} H_{0, i}^{\lambda} y$. However, $y_{\Omega}$ is a linear function of the state components associated to $\varphi_{e}\left(\mathbf{V}_{\mathbf{Y}}\right)$, which are not necessarily included in $\bar{\varphi}\left(\mathbf{V}_{\mathbf{0}}\right)$. Thus, the components of $\varphi_{e}\left(\mathbf{V}_{\mathbf{Y}}\right) \backslash\left(\bar{\varphi}\left(\mathbf{V}_{\mathbf{0}}\right) \cap \varphi_{e}\left(\mathbf{V}_{\mathbf{Y}}\right)\right)$ must be added to the components which must be expressed using $y_{\Omega}$. The number of state variables we can express using $y_{\Omega}$ is equal to $\theta\left(\mathbf{V}_{\mathbf{Y}}\right)$. Thus, $\mathbf{V}_{\mathbf{Y}}$ must satisfy $\theta\left(\mathbf{V}_{\mathbf{Y}}\right) \geq \operatorname{card}\left(\varphi_{e}\left(\mathbf{V}_{\mathbf{Y}}\right) \cup \bar{\varphi}\left(\mathbf{V}_{\mathbf{0}}\right)\right)-k+1-\operatorname{card}\left(\bar{\varphi}\left(\mathbf{V}_{\mathbf{0}}\right) \cap \mathbf{V}_{\mathbf{X} \mathbf{U}^{\mathbf{0}}}^{*}\right)=n_{1}$.

Furthermore, assume now that there exists a vertex subset $\mathbf{V}_{\mathbf{Y}}^{\prime} \subseteq \mathbf{Y}$ satisfying, $\theta\left(\mathbf{V}_{\mathbf{Y}}^{\prime}\right)=n_{1}^{\prime}>n_{1}$.

The latter inequality implies that $\theta\left(\mathbf{V}_{\mathbf{Y}}^{\prime}\right) \geq \operatorname{card}\left(\varphi_{e}\left(\mathbf{V}_{\mathbf{Y}}^{\prime}\right) \cup \bar{\varphi}\left(\mathbf{V}_{\mathbf{0}}\right)\right)-(k-1)+1-\operatorname{card}\left(\bar{\varphi}\left(\mathbf{V}_{\mathbf{0}}\right) \cap \mathbf{V}_{\mathbf{X}}^{*} \mathbf{U}^{\mathbf{0}}\right)$. Thus, that for all $\mathbf{V}_{\mathbf{0}}^{\prime} \subset \mathbf{V}_{\mathbf{0}}$ such that $\operatorname{card}\left(\mathbf{V}_{\mathbf{0}}^{\prime}\right)=\operatorname{card}\left(\mathbf{V}_{\mathbf{0}}\right)-1=k-1$, we have $\theta\left(\mathbf{V}_{\mathbf{Y}}^{\prime}\right) \geq \operatorname{card}\left(\varphi_{e}\left(\mathbf{V}_{\mathbf{Y}}^{\prime}\right) \cup \bar{\varphi}\left(\mathbf{V}_{\mathbf{0}}\right)\right)-(k-1)-\operatorname{card}\left(\bar{\varphi}\left(\mathbf{V}_{\mathbf{0}}^{\prime}\right) \cap \mathbf{V}_{\mathbf{X U ^ { 0 }}}^{*}\right)+1$. According to the sufficiency of Lemma 2 proved above $\pi\left(\mathbf{V}_{\mathbf{0}}^{\prime}\right)$ is useful (see Remark 2). However, we take as hypothesis $\nexists \mathbf{V}_{\mathbf{0}}^{\prime} \subset \mathbf{V}_{\mathbf{0}}$ such that $\pi\left(\mathbf{V}_{\mathbf{0}}^{\prime}\right)$ is useful. Consequently, $\nexists \mathbf{Z} \mathbf{V}_{\mathbf{Y}}^{\prime} \subseteq \mathbf{Y}$ such that $\theta\left(\mathbf{V}_{\mathbf{Y}}^{\prime}\right) \leq n_{1}$.

On the one hand, we have proved that the existence of a vector $P_{k}^{\lambda} \in \mathbb{R}^{1 \times k}$, such that $P_{k}^{\lambda}\left(z_{i_{1}}, z_{i_{2}}, \ldots, z_{i_{k}}\right)^{T}$ can be expressed in function of $\dot{y}$ and $u_{i} y$ implies the existence of $\mathbf{V}_{\mathbf{Y}} \subseteq \mathbf{Y}$ such that $\theta\left(\mathbf{V}_{\mathbf{Y}}\right) \geq n_{1}$. On the other hand, the hypothesis of lemma 1 imposes that there cannot exist $\mathbf{V}_{\mathbf{Y}} \subseteq \mathbf{Y}$ such that $\theta\left(\mathbf{V}_{\mathbf{Y}}\right)>n_{1}$. Consequently, the existence of a vector $P_{k}^{\lambda} \in \mathbb{R}^{1 \times k}$ such that $P_{k}^{\lambda}\left(z_{i_{1}}, z_{i_{2}}, \ldots, z_{i_{k}}\right)^{T}$ can be expressed in function of $\dot{y}$ and $u_{i} y$ implies the existence of $\mathbf{V}_{\mathbf{Y}} \subseteq \mathbf{Y}$ such that $\theta\left(\mathbf{V}_{\mathbf{Y}}\right)=n_{1}=\operatorname{card}\left(\varphi_{e}\left(\mathbf{V}_{\mathbf{Y}}\right) \cup \bar{\varphi}\left(\mathbf{V}_{\mathbf{0}}\right)\right)-k-\operatorname{card}\left(\bar{\varphi}\left(\mathbf{V}_{\mathbf{0}}\right) \cap \mathbf{V}_{\mathbf{X U}^{\mathbf{0}}}^{*}\right)+1$.

The hypothesis of Lemma 2, which is in relation with the notion of structural minimality, is necessary to ensure that the combined vertices verifying conditions of Lemma 2 represent effectively the number of linear independent vectors $P^{\lambda}$ such that $P^{\lambda} z$ can be expressed in function of $\dot{y}$ and $u_{i} y$. Indeed, assume that $\mathbf{z}_{1}$ is useful and that $\left\{\mathbf{z}_{1}, \mathbf{z}_{2}\right\}$ satisfies condition (17). We have then two cases: either $\mathbf{z}_{2}$ is useful and in this case the vectors representing $\left\{\mathbf{z}_{1}\right\},\left\{\mathbf{z}_{2}\right\},\left\{\mathbf{z}_{1}, \mathbf{z}_{2}\right\}$ are 
not linearly independent. Or $\mathbf{z}_{2}$ is not useful and so only $P_{1}^{\lambda}$ representing $\mathbf{z}_{1}$ is such that $P_{1}^{\lambda} z$ can be expressed in function of $\dot{y}$ and $u_{i} y$. In both cases, the fact that $\left\{\mathbf{z}_{1}, \mathbf{z}_{2}\right\}$ satisfies condition (17) is not significative in the search of useful vertices corresponding to vectors $P^{\lambda}$ such that $P^{\lambda} z$ can be expressed in function of $\dot{y}$ and $u_{i} y$.

Let us denote by $\mathbf{Z}^{\mathbf{1}}$ the biggest (in the sens of cardinality) structurally minimal set of useful vertices of $\mathbf{Z}_{\mathbf{C}}$ obtained after the first step. Since this set is structurally minimal, it represents all the linearly independent combinations of the first derivative of the output components. $\mathbf{Z}^{\mathbf{1}}$ represents then $\Gamma_{0}^{\lambda} \dot{Y}_{0}-\sum_{i=1}^{m} u_{i} H_{0, i}^{\lambda} Y_{0}=C_{1}^{\lambda} x$ and $\mathbf{Y} \cup \mathbf{Z}^{\mathbf{1}}$ represents $Y_{1}$ in relations (4).

If $\mathbf{Z}^{\mathbf{1}} \neq \emptyset$ then the first derivative of the output components can be exploited to give new informations on the state components for any value of $u$. If $\theta\left(\mathbf{Y} \cup \mathbf{Z}^{\mathbf{1}}\right)=\operatorname{card}(\mathbf{X})=n$ then system $\left(\Sigma_{\Lambda}\right)$ is uniformly observable. Otherwise, the output must be differentiated once again. At this aim, a second step is necessary to determinate which vertices of $\mathbf{Z}_{\mathbf{C}}$ are useful for a second differentiation of the output.

In this second step, we consider $\mathbf{Y} \cup \mathbf{Z}^{\mathbf{1}}$ as the new output vertices of the system. This is equivalent in form (4), to the fact that in the second step we differentiate $Y_{1} \stackrel{\text { def }}{=}\left(\begin{array}{l}C^{\lambda} \\ C_{1}^{\lambda}\end{array}\right) x$ to obtain a relation on the form $\Gamma_{1}^{\lambda} \dot{Y}_{1}=C_{2}^{\lambda} x+\sum_{i=1}^{m} u_{i} H_{1, i}^{\lambda} Y_{1}$. As a matter of fact, this second step is similar to the first one but with considering as an output $Y_{1}$ instead of $Y_{0}=y$. Graphically, this amounts to consider $\mathbf{Y} \cup \mathbf{Z}^{\mathbf{1}}$ as output vertex subset instead of $\mathbf{Y}$.

Therefore, in this second step, we can follow a similar approach than the one presented previously in Lemma 2 by replacing " $\exists \mathbf{V}_{\mathbf{Y}} \subseteq \mathbf{Y}$ "by " $\exists \mathbf{V}_{\mathbf{Y}} \subseteq \mathbf{Y}$ and a structurally minimal subset $\mathbf{V}_{\mathbf{Z}} \subseteq \wp\left(\mathbf{Z}^{1}\right) ", \mathbf{V}_{\mathbf{M}^{0}}^{*}$ by $\mathbf{V}_{\mathbf{M}^{1}}^{*}, \mathbf{V}_{\mathbf{X}^{0}}^{*}$ by $\mathbf{V}_{\mathbf{X}^{1}}^{*}$ and $\mathbf{V}_{\mathbf{X U}^{0}}^{*}$ by $\mathbf{V}_{\mathbf{X} \mathbf{U}^{1}}^{*}$, where $\mathbf{V}_{\mathbf{M}^{\mathbf{i}}}^{*}, \mathbf{V}_{\mathbf{X}^{\mathbf{i}}}^{*}$ and $\mathbf{V}_{\mathbf{X} \mathbf{U}^{\mathbf{i}}}^{*}$ for $i \geq 0$ are defined by:

Definition 3 For $i \geq 1, \mathbf{V}_{\mathbf{M}^{\mathbf{i}}}^{*}$ is the maximal (in the sens of the cardinality) subset of $\mathbf{Y} \cup \wp\left(\mathbf{Z}^{\mathbf{i}}\right)$ such that: $\mathbf{V}_{\mathbf{Z}} \stackrel{\text { def }}{=} \mathbf{V}_{\mathbf{M}^{\mathbf{i}}}^{*} \cap \mathbf{Z}_{\mathbf{C}}$ is structurally minimal and $\theta\left(\mathbf{V}_{\mathbf{M}^{\mathbf{i}}}^{*}\right)=\operatorname{card}\left(\varphi_{e}\left(\mathbf{V}_{\mathbf{M}^{\mathbf{i}}}^{*}\right)\right)$. We take the following notations: $\mathbf{V}_{\mathbf{X}^{\mathbf{i}}}^{*}=\varphi_{e}\left(\mathbf{V}_{\mathbf{M}^{\mathbf{i}}}^{*}\right)$ and $\mathbf{V}_{\mathbf{X U}^{\mathbf{i}}}^{*}=\left\{\mathbf{s} \in \mathbf{X U}\right.$ such that,$\left.\alpha(\{\mathbf{s}\}) \subseteq \mathbf{V}_{\mathbf{X}^{\mathbf{i}}}^{*}\right\}$.

Let us denote by $\mathbf{Z}^{2}$ the set of useful vertices representing linearly independent combinations of the first and second derivatives of the output components. If $\theta\left(\mathbf{Y} \cup \mathbf{Z}^{2}\right)=\operatorname{card}(\mathbf{X})=n$ then system $\left(\Sigma_{\Lambda}\right)$ is generically uniform observable. Otherwise, we must differentiate the output once again and so on. We present the algorithm which allows us to determine the set $\mathbf{Z}^{*}$ which is the limit of the structurally minimal subset sequence $\left(\mathbf{Z}^{\mathbf{i}}\right)$ and which represents the set of all useful vertices:

\section{Algorithm 1}


Initialisation : $\mathbf{Z}^{\mathbf{0}}=\emptyset$ and $i=0$

Step i: $\mathbf{Q}_{\mathbf{i}}=\varphi\left(\varphi_{e}\left(\mathbf{Y} \cup \mathbf{Z}^{\mathbf{0}}\right)\right), \mathbf{Z}^{\mathbf{i}+\mathbf{1}}=\emptyset$. Compute $\mathbf{V}_{\mathbf{X} \mathbf{U}^{\mathbf{i}}}^{*}$ according to Definition 3

\section{Determination of the useful vertices:}

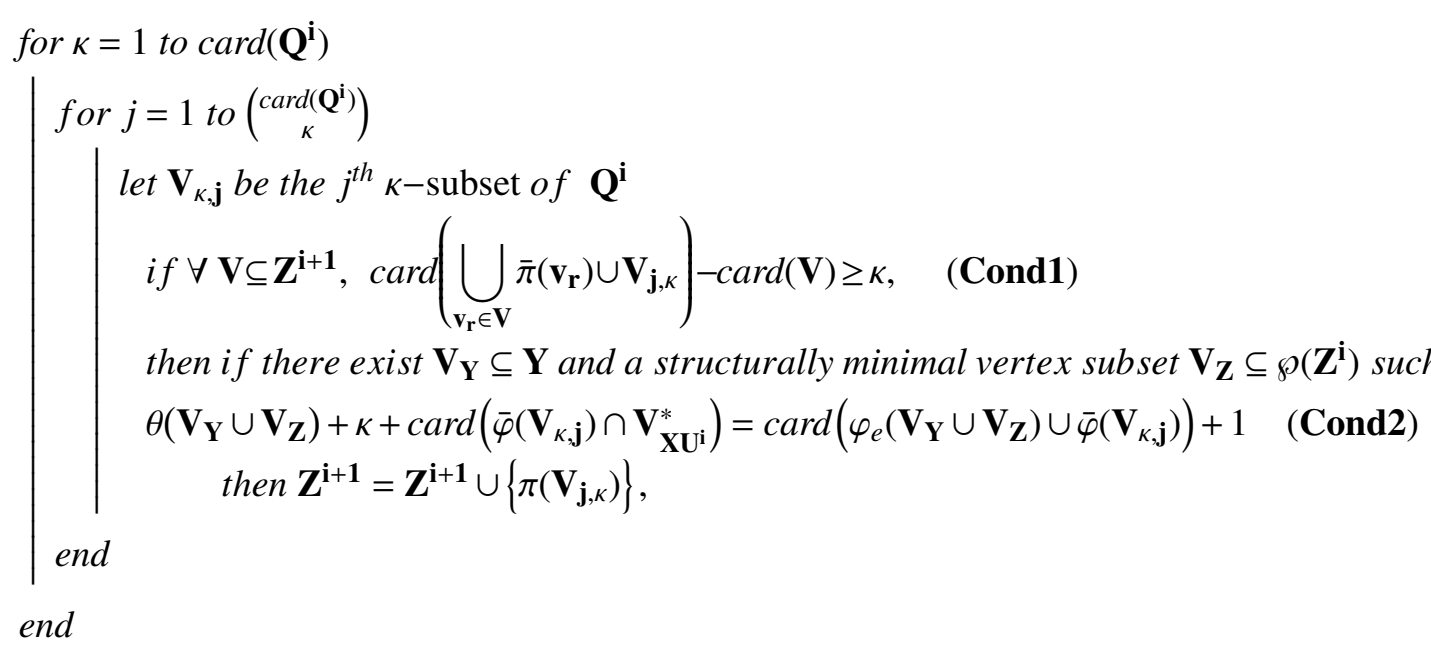

End of step $i$ : If $\theta\left(\mathbf{Z}^{\mathbf{i}+\mathbf{1}}\right) \neq \theta\left(\mathbf{Z}^{\mathbf{i}}\right)$, we must differentiate y again: $i \leftarrow i+1$ and we go to Step $\boldsymbol{i}$ Else $\mathbf{Z}^{*}=\mathbf{Z}^{\mathbf{i}}$

\section{Remark 3}

- Condition (Cond1) above ensures that $\mathbf{Z}^{\mathbf{i}+\mathbf{1}}$ is structurally minimal. Due to condition (Cond1), if $\mathbf{V}_{\mathbf{0}} \subseteq \mathbf{Z}$ satisfies condition (Cond2) then either $\pi\left(\mathbf{V}_{\mathbf{0}}\right)$ belongs to $\mathbf{Z}^{\mathbf{i}+\mathbf{1}}$ or $\pi\left(\mathbf{V}_{\mathbf{0}}\right) \cup \mathbf{Z}^{\mathbf{i}+\mathbf{1}}$ is not structurally minimal. This means that if $\mathbf{V}_{\mathbf{0}} \subseteq \mathbf{Z}$ satisfies condition (Cond2) then $\exists \overline{\mathbf{V}}_{\mathbf{0}} \subseteq \mathbf{V}_{\mathbf{0}}$ such that $\pi\left(\overline{\mathbf{V}}_{\mathbf{0}}\right) \in \wp\left(\mathbf{Z}^{\mathbf{i}+\mathbf{1}}\right)$. Furthermore, by construction of $\wp\left(\mathbf{Z}^{\mathbf{i}+\mathbf{1}}\right), \forall \mathbf{s} \in \wp\left(\mathbf{Z}^{\mathbf{i}+\mathbf{1}}\right), \bar{\pi}(\mathbf{s})$ satisfies condition (Cond2).

- It is easy to see from condition (Cond2) that for $i \geq 0$, if $\mathbf{s} \in \mathbf{Z}^{\mathbf{i}}$ then there exists $\mathbf{s}_{\mathbf{0}}$ such that $\bar{\pi}\left(\mathbf{s}_{\mathbf{0}}\right) \subseteq \bar{\pi}(\mathbf{s})$ and $\mathbf{s}_{\mathbf{0}} \in \mathbf{Z}^{\mathbf{i}+\mathbf{1}}$.

We illustrate hereafter the previous algorithm on a simple example:

Example 2 Consider the structured system defined by:

$A^{\lambda}=\left(\begin{array}{cccc}\lambda_{1} & 0 & \lambda_{2} & 0 \\ 0 & \lambda_{3} & 0 & 0 \\ 0 & 0 & 0 & \lambda_{4} \\ 0 & 0 & 0 & \lambda_{5}\end{array}\right), B_{1}^{\lambda}=\left(\begin{array}{cccc}0 & 0 & \lambda_{6} & 0 \\ 0 & \lambda_{7} & \lambda_{8} & 0 \\ 0 & 0 & 0 & 0 \\ 0 & 0 & 0 & 0\end{array}\right)$ and $C^{\lambda}=\left(\begin{array}{cccc}\lambda_{9} & \lambda_{10} & 0 & 0 \\ 0 & \lambda_{11} & 0 & 0\end{array}\right)$. To such a model, we associate the digraph in Figure 2.

For such a system, we apply algorithm 1 :

Step 0: Since $\theta(\mathbf{Y})=2$ and $\varphi_{e}(\mathbf{Y})=\left\{\mathbf{x}_{\mathbf{1}}, \mathbf{x}_{\mathbf{2}}\right\}$ we have that $\mathbf{V}_{\mathbf{M}^{\mathbf{0}}}^{*}=\mathbf{Y}=\left\{\mathbf{y}_{\mathbf{1}}, \mathbf{y}_{\mathbf{2}}\right\}$ satisfies condition (15). So, we deduce $\mathbf{V}_{\mathbf{X}^{0}}^{*}=\varphi\left(\mathbf{V}_{\mathbf{M}^{0}}^{*}\right)=\left\{\mathbf{x}_{\mathbf{1}}, \mathbf{x}_{\mathbf{2}}\right\}$ and $\mathbf{V}_{\mathbf{X U}^{\mathbf{0}}}^{*}=\left\{\mathbf{x}_{\mathbf{2}} \mathbf{u}_{\mathbf{1}}\right\}$. $\mathbf{Q}_{\mathbf{0}}=\left\{\mathbf{z}_{\mathbf{1}}, \mathbf{z}_{\mathbf{2}}\right\}$.

For $\mathbf{V}_{\mathbf{1}, \mathbf{1}}=\left\{\mathbf{z}_{\mathbf{1}}\right\}$, there does not exist any subset $\mathbf{V}_{\mathbf{Y}}$ of $\mathbf{Y}\left(\mathbf{Z}^{\mathbf{0}}=\emptyset\right)$ which allows to satisfy equality (Cond2). Indeed, for $\mathbf{V}_{\mathbf{Y}}=\left\{\mathbf{y}_{\mathbf{1}}\right\}, \theta\left(\mathbf{V}_{\mathbf{Y}}\right)+\kappa+\operatorname{card}\left(\bar{\varphi}\left(\mathbf{V}_{\mathbf{1}, \mathbf{1}}\right) \cap \mathbf{V}_{\mathbf{X} \mathbf{U}^{\mathbf{0}}}^{*}\right)=1+1+0=2$ and 


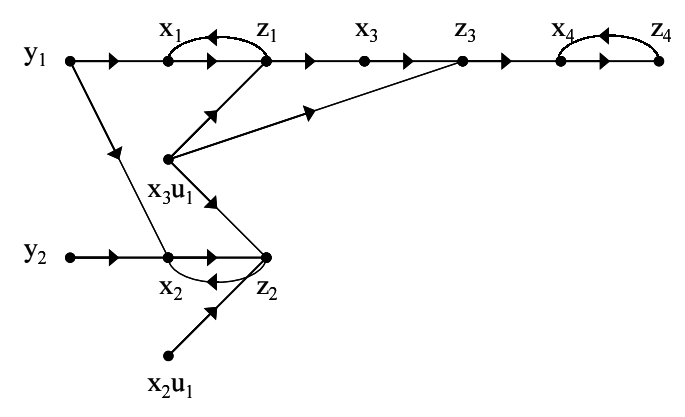

Figure 2: Digraph associated to Example 2

$\operatorname{card}\left(\varphi_{e}\left(\mathbf{V}_{\mathbf{Y}}\right) \cup \bar{\varphi}\left(\mathbf{V}_{\mathbf{1}, \mathbf{1}}\right)\right)+1=3+1=4$. This is also the case for $\mathbf{V}_{\mathbf{Y}}=\left\{\mathbf{y}_{\mathbf{2}}\right\}$ where $\theta\left(\mathbf{V}_{\mathbf{Y}}\right)+\kappa+$ $\operatorname{card}\left(\bar{\varphi}\left(\mathbf{V}_{\mathbf{1}, \mathbf{1}}\right) \cap \mathbf{V}_{\mathbf{X U}}^{*}\right)=1+1+0=2$ and $\operatorname{card}\left(\varphi_{e}\left(\mathbf{V}_{\mathbf{Y}}\right) \cup \bar{\varphi}\left(\mathbf{V}_{\mathbf{1}, \mathbf{1}}\right)\right)+1=3+1=4$ and for $\mathbf{V}_{\mathbf{Y}}=$ $\left\{\mathbf{y}_{\mathbf{1}}, \mathbf{y}_{\mathbf{2}}\right\}$ where $\theta\left(\mathbf{V}_{\mathbf{Y}}\right)+\kappa+\operatorname{card}\left(\bar{\varphi}\left(\mathbf{V}_{\mathbf{1}, \mathbf{1}}\right) \cap \mathbf{V}_{\mathbf{X} \mathbf{U}^{\mathbf{0}}}^{*}\right)=2+1+0=3$ and $\operatorname{card}\left(\varphi_{e}\left(\mathbf{V}_{\mathbf{Y}}\right) \cup \bar{\varphi}\left(\mathbf{V}_{\mathbf{1}, \mathbf{1}}\right)\right)+1=$ $3+1=4$.

We can do exactly the same computations to conclude that for $\mathbf{V}_{\mathbf{1 , 2}}=\left\{\mathbf{z}_{\mathbf{2}}\right\}$, there does not exist any subset $\mathbf{V}_{\mathbf{Y}}$ of $\mathbf{Y}$ which allows to satisfy equality (Cond2). On the other hand, for $\mathbf{V}_{\mathbf{2}, \mathbf{1}}=\left\{\mathbf{z}_{\mathbf{2}}, \mathbf{z}_{\mathbf{2}}\right\}$, if we take $\mathbf{V}_{\mathbf{Y}}=\left\{\mathbf{y}_{\mathbf{1}}, \mathbf{y}_{\mathbf{2}}\right\}$, condition (Cond2) is verified: $\theta\left(\mathbf{V}_{\mathbf{Y}}\right)+\kappa+\operatorname{card}\left(\bar{\varphi}\left(\mathbf{V}_{\mathbf{2}, \mathbf{1}}\right) \cap \mathbf{V}_{\mathbf{X U}^{\mathbf{0}}}^{*}\right)=2+2+1=$ 5 and $\operatorname{card}\left(\varphi_{e}\left(\mathbf{V}_{\mathbf{Y}}\right) \cup \bar{\varphi}\left(\mathbf{V}_{\mathbf{2}, \mathbf{1}}\right)\right)+1=4+1=5$.

Thus, $\mathbf{Z}^{\mathbf{1}}=\left\{\mathbf{z}_{\mathbf{1}, \mathbf{2}}\right\}$. As $\theta\left(\mathbf{Z}^{\mathbf{1}}\right)=1>\theta\left(\mathbf{Z}^{\mathbf{0}}\right)=0$, we go to step 1 .

Step 1: Since, $\theta\left(\mathbf{Z}^{\mathbf{1}} \cup \mathbf{Y}\right)=3$ and $\operatorname{card}\left(\varphi_{e}\left(\mathbf{Z}^{\mathbf{1}} \cup \mathbf{Y}\right)\right)=\operatorname{card}\left(\left\{\mathbf{x}_{\mathbf{1}}, \mathbf{x}_{\mathbf{2}}, \mathbf{x}_{\mathbf{3}}\right\}\right)=3$, we have that $\mathbf{V}_{\mathbf{X}^{1}}^{*}=$ $\left\{\mathbf{x}_{1}, \mathbf{x}_{\mathbf{2}}, \mathbf{x}_{\mathbf{3}}\right\}, \mathbf{V}_{\mathbf{X U}^{1}}^{*}=\alpha\left(\mathbf{V}_{\mathbf{X}^{1}}^{*}\right)=\left\{\mathbf{x}_{\mathbf{2}} \mathbf{u}_{\mathbf{1}}, \mathbf{x}_{\mathbf{3}} \mathbf{u}_{\mathbf{1}}\right\}$. Furthermore, $\mathbf{Q}_{\mathbf{1}}=\left\{\mathbf{z}_{\mathbf{1}}, \mathbf{z}_{\mathbf{2}}, \mathbf{z}_{\mathbf{3}}\right\}$ and for each one-subset of $\mathbf{Q}_{\mathbf{1}}$ there exist $\mathbf{V}_{\mathbf{Y}} \subseteq \mathbf{Y}$ and a structurally minimal vertex subset $\mathbf{V}_{\mathbf{Z}} \subseteq \wp\left(\mathbf{Z}^{\mathbf{1}}\right)$ such that equality (Cond2) is satisfied:

- For $\mathbf{V}_{\mathbf{1}, \mathbf{1}}=\left\{\mathbf{z}_{\mathbf{1}}\right\}$, if we take $\mathbf{V}_{\mathbf{Y}}=\left\{\mathbf{y}_{\mathbf{1}}, \mathbf{y}_{\mathbf{2}}\right\}$ and $\mathbf{V}_{\mathbf{Z}}=\emptyset$, we have $\theta\left(\mathbf{V}_{\mathbf{Y}} \cup \mathbf{V}_{\mathbf{Z}}\right)+\kappa+$ $\operatorname{card}\left(\bar{\varphi}\left(\mathbf{V}_{\mathbf{1 , 1}}\right) \cap \mathbf{V}_{\mathbf{X} \mathbf{U}^{1}}^{*}\right)=2+1+1=4$ and $\operatorname{card}\left(\varphi_{e}\left(\mathbf{V}_{\mathbf{Y}} \cup \mathbf{V}_{\mathbf{Z}}\right) \cup \bar{\varphi}\left(\mathbf{V}_{\mathbf{1}, \mathbf{1}}\right)\right)+1=3+1=4$.

- For $\mathbf{V}_{\mathbf{1}, \mathbf{2}}=\left\{\mathbf{z}_{\mathbf{2}}\right\}$, if we take $\mathbf{V}_{\mathbf{Y}}=\left\{\mathbf{y}_{\mathbf{2}}\right\}$ and $\mathbf{V}_{\mathbf{Z}}=\emptyset$, we have $\theta\left(\mathbf{V}_{\mathbf{Y}} \cup \mathbf{V}_{\mathbf{Z}}\right)+\kappa+$ $\operatorname{card}\left(\bar{\varphi}\left(\mathbf{V}_{\mathbf{1}, \mathbf{1}}\right) \cap \mathbf{V}_{\mathbf{X} \mathbf{U}^{1}}^{*}\right)=1+1+2=4 \operatorname{and} \operatorname{card}\left(\varphi_{e}\left(\mathbf{V}_{\mathbf{Y}} \cup \mathbf{V}_{\mathbf{Z}}\right) \cup \bar{\varphi}\left(\mathbf{V}_{\mathbf{1}, \mathbf{1}}\right)\right)+1=3+1=3$.

- For $\mathbf{V}_{\mathbf{1 , 3}}=\left\{\mathbf{z}_{\mathbf{3}}\right\}$ if we take $\mathbf{V}_{\mathbf{Y}}=\left\{\mathbf{y}_{\mathbf{1}}, \mathbf{y}_{\mathbf{2}}\right\}$ and $\mathbf{V}_{\mathbf{Z}}=\left\{\mathbf{z}_{\mathbf{1}, \mathbf{2}}\right\}\left(\mathbf{V}_{\mathbf{Z}}\right.$ is obviously structurally minimal and is included in $\left.\mathbf{Z}^{\mathbf{1}}\right)$, we have $\theta\left(\mathbf{V}_{\mathbf{Y}} \cup \mathbf{V}_{\mathbf{Z}}\right)+\kappa+\operatorname{card}\left(\bar{\varphi}\left(\mathbf{V}_{\mathbf{1}, \mathbf{1}}\right) \cap \mathbf{V}_{\mathbf{X} \mathbf{U}^{1}}^{*}\right)=3+1+1=5$ and $\operatorname{card}\left(\varphi_{e}\left(\mathbf{V}_{\mathbf{Y}} \cup \mathbf{V}_{\mathbf{Z}}\right) \cup \bar{\varphi}\left(\mathbf{V}_{\mathbf{1}, \mathbf{1}}\right)\right)+1=4+1=5$.

Moreover, since all the other subsets of $\mathbf{Q}_{\mathbf{1}}$ do not satisfy condition (Cond1) because for any $\mathbf{V}_{\kappa, \mathbf{j}} \subseteq \mathbf{Q}_{\mathbf{1}}, \kappa \geq 2,\left\{\pi\left(\mathbf{V}_{\kappa, \mathbf{j}}\right)\right\} \cup\left\{\mathbf{z}_{\mathbf{1}}, \mathbf{z}_{\mathbf{2}}, \mathbf{z}_{\mathbf{3}}\right\}$ is not structurally minimal. Thus, $\mathbf{Z}^{\mathbf{2}}=\left\{\mathbf{z}_{\mathbf{1}}, \mathbf{z}_{\mathbf{2}}, \mathbf{z}_{\mathbf{3}}\right\}$.

Let us now do some comments about algorithm 1. At step 0, $\mathbf{Y}$ represents all output derivatives of order 0 exploitable to reconstruct a part of the state for any value of input value $u$. Indeed relation $y=C^{\lambda} x$ does not depend on input value $u$. We use Lemma 2 to compute $\mathbf{Z}^{1}$ which 
represents all vertices of $\mathbf{Z}_{\mathbf{C}}$ associated to all the linearly independent combinations of dynamic variables which can be expressed in function of the output, its first derivative and the input: $P^{\lambda} z=$ $\Gamma_{0}^{\lambda} \dot{Y}_{0}+\sum_{i=1}^{m} u_{i} H_{0, i}^{\lambda} Y_{0}=P^{\lambda} A^{\lambda} x$. This equation allows us to "observe" $P^{\lambda} A^{\lambda} x$ for any value $u$. The linear independence of such combinations is ensured by condition (Cond1) which imposes to $\mathbf{Z}^{\mathbf{1}}$ to be structurally minimal and which ensures that the hypothesis of Lemma 2 is satisfied. $\mathbf{Z}^{\mathbf{1}}$ represents then $\Gamma_{0}^{\lambda} \dot{Y}_{0}-\sum_{i=1}^{m} u_{i} H_{0, i}^{\lambda} Y_{0}=C_{1}^{\lambda} x$ defined in relations (4) and $\mathbf{Y} \cup \mathbf{Z}^{\mathbf{1}}$ corresponds to $Y_{1}$. Step $i$ of the algorithm is equivalent to step 0 by enlarging the output components to $Y_{i}$ and so the vertex set $\mathbf{Y}$ to $\mathbf{Y} \cup \mathbf{Z}^{\mathbf{i}}$ or, roughly speaking, to all the part of state vector observed in the previous steps. Indeed, in form (4), Step $i$ corresponds to the search of matrix $\Gamma_{i}$ such that: $\Gamma_{i}^{\lambda} \dot{Y}_{i}=$ $C_{i+1}^{\lambda} x+\sum_{j=1}^{m} u_{i} H_{i, j}^{\lambda} Y_{i}$, where $Y_{i} \stackrel{\text { def }}{=}\left(\begin{array}{l}C^{\lambda} \\ C_{i}^{\lambda}\end{array}\right) x$. This step is equivalent to step 0 by considering that the output vector is enlarged to $Y_{i}$. So, as in step 0 , we mark in step $i$ all the vertices of $\mathbf{Z}_{\mathbf{C}}$ which correspond to linear independent combinations $P^{\lambda} z$ satisfying relation of the form (10) between the first derivative of the variables associated to $\mathbf{Y} \cup \mathbf{Z}^{\mathbf{i}}$, corresponding to vector $Y_{i}$ in (4), the input and the state. Consequently, edges starting from $\mathbf{Y} \cup \mathbf{Z}^{\mathbf{i}}$ correspond to all relations, linking the state to the input and the output derivatives of order less or equal to $i+1$, which allow us to observe a part of the state for any input value. Due to the second item of Remark 3, $\mathbf{Z}^{\mathbf{i}}$ is a non-decreasing sequence of structurally minimal subset w.r.t. the function $\theta\left(\theta\left(\mathbf{Z}^{\mathbf{i}}\right) \leq \theta\left(\mathbf{Z}^{\mathbf{i}+\mathbf{1}}\right)\right)$. Moreover, since $\theta\left(\mathbf{Z}^{\mathbf{i}}\right)$ is bounded by $n$, there exists an integer $k$ for which $\theta\left(\mathbf{Z}^{\mathbf{k}+\mathbf{j}}\right)=\theta\left(\mathbf{Z}^{\mathbf{k}}\right)$, for all $j \geq 0$. So after a finite number of iterations the algorithm stops when $\theta\left(\mathbf{Z}^{\mathbf{i}+\mathbf{1}}\right)=\theta\left(\mathbf{Z}^{\mathbf{i}}\right)$ which means that in the $(i+1)^{\text {th }}$ step, the $(i+1)^{\text {th }}$ output differentiation does not give any new relation useful to achieve uniform observability of $\left(\Sigma_{\Lambda}\right)$. In this case, we pose $\mathbf{Z}^{*}=\mathbf{Z}^{\mathbf{k}}$ and vertex subset $\mathbf{Y} \cup \mathbf{Z}^{*}$, represents all the vertices of $\mathbf{Y} \cup \mathbf{Z}_{\mathbf{C}}$ which can be associated to output vector $Y_{k}=\left(\begin{array}{c}C \\ C_{k}\end{array}\right) x$ of relation (4). These vertices can be associated to useful and independent relations between the outputs and their derivatives and the state and which are exploitable to observe $x$ for any input value. The system can be put into the form (4) if and only if $\theta\left(\mathbf{Y} \cup \mathbf{Z}^{*}\right)=n$ which is equivalent, according to the construction of $\mathbf{Z}^{*}$, to g_rank $\left(\begin{array}{c}C^{\lambda} \\ C_{k}^{\lambda}\end{array}\right)=n$.

\subsection{Graphic conditions for generic uniform observability}

The first part of this section suggests immediately the following proposition :

Proposition 2 Consider structured bilinear system $\left(\Sigma_{\Lambda}\right)$ represented by digraph $\mathcal{G}\left(\Sigma_{\Lambda}\right)$. Structured system $\left(\Sigma_{\Lambda}\right)$ can be put into form (4) if and only if in graph $\mathcal{G}\left(\Sigma_{\Lambda}\right), \theta\left(\mathbf{Y} \cup \mathbf{Z}^{*}\right)=n$, where $\mathbf{Z}^{*}$ is computed according to Algorithm 1. 
Proof: The proof of $(1) \Leftrightarrow(2)$ is immediate using Lemmas 1 and 2, algorithm 1 and Property P1 of function $\theta$.

Note that if the system can be put into form (4), then there exists an integer $k$ such that $\mathbf{V}_{\mathbf{X}^{\mathbf{k}}}^{*}=\mathbf{X}$, and $\mathbf{V}_{\mathbf{X} \mathbf{U}^{\mathbf{k}}}^{*}=\mathbf{X U}$. In this case, $\mathbf{Z}^{\mathbf{k}+\mathbf{1}}=\mathbf{Z}^{*}=\mathbf{Z}$. Thus, from Proposition 2, we can deduce the following corollary which represents the main result of the paper:

Corollary 1 Consider structured bilinear system $\left(\Sigma_{\Lambda}\right)$ represented by digraph $\mathcal{G}\left(\Sigma_{\Lambda}\right)$.

(1) $\left(\Sigma_{\Lambda}\right)$ is generically uniform observable only if, in graph $\mathcal{G}\left(\Sigma_{\Lambda}\right)$, there exists a path from $\mathbf{Y}$ to each state vertex and there exists a $v$-disjoint subgraph $\mathcal{S}_{\mathcal{G}}$ which covers all the state vertices.

(2) $\left(\Sigma_{\Lambda}\right)$ is generically uniform observable if $\mathbf{Z}^{*}=\mathbf{Z}$, where $\mathbf{Z}^{*}$ is computed using to Algorithm 1 .

Note that the condition (1) of Corollary 1 is necessary because it means also the observability of the generic observability of the system for $u(t)=0$. Furthermore, from the results of [9], condition (2) is obviously necessary and sufficient in the single output case.

The results of Corollary 1 and Algorithm 1 (used to compute $\mathbf{Z}^{*}$ ) show that to achieve the uniform observability, we can either add sensors or put some inputs to zero by removing some actuators. The first solution is obviously more suitable. In the one hand it allows to enlarge $\mathbf{Z}^{*}$ and to achieve the first condition of Corollary 1 , while the second one allows to enlarge $\mathbf{Z}^{*}$ only. On the second hand, the second solution is not always acceptable from a control point of view.

\section{Example}

In this section, we illustrate the results presented above with an example. The latter is intentionally not very complicated. Nevertheless, it is clear that the algorithm we propose is well suited to largescale systems using combinatorial programming techniques.

Example 3 Consider the SBLS represented in the digraph of Figure 3. This system has 14 state components. The matrix representation of this system is not given because of lack of space. Nevertheless, it can be easily deduced from the digraph. First, note that necessary sufficient condition (1) enounced in Corollary 1 is satisfied as it is shown by the v-disjoint subgraph (Figure 4) which covers all the state vertices.

We use now algorithm 1 to determine $\mathbf{Z}^{*}$ :

Step 0: First, we compute $\mathbf{V}_{\mathbf{M}^{0}}^{*}=\left\{\mathbf{y}_{\mathbf{3}}, \mathbf{y}_{\mathbf{5}}\right\}, \mathbf{V}_{\mathbf{X}^{0}}^{*}=\left\{\mathbf{x}_{\mathbf{4}}, \mathbf{x}_{\mathbf{8}}\right\}, \mathbf{V}_{\mathbf{X U}^{0}}^{*}=\left\{\mathbf{x}_{\mathbf{4}} \mathbf{u}_{\mathbf{1}}, \mathbf{x}_{\mathbf{4}} \mathbf{u}_{\mathbf{2}}\right\}$ and graphically we have $\mathbf{Q}^{\mathbf{0}}=\left\{\mathbf{z}_{\mathbf{1}}, \mathbf{z}_{\mathbf{2}}, \mathbf{z}_{\mathbf{3}}, \mathbf{z}_{\mathbf{4}}, \mathbf{z}_{\mathbf{5}}, \mathbf{z}_{\mathbf{6}}, \mathbf{z}_{7}, \mathbf{z}_{\mathbf{8}}\right\}$. Because of the presence of $\mathbf{x}_{\mathbf{1}} \mathbf{u}_{\mathbf{1}}$ in $\bar{\varphi}\left(\left\{\mathbf{z}_{\mathbf{2}}\right\}\right)$ and the fact that $\varphi_{e}\left(\left\{\mathbf{y}_{\mathbf{1}}, \mathbf{y}_{\mathbf{2}}\right\}\right)=\left\{\mathbf{x}_{\mathbf{1}}, \mathbf{x}_{\mathbf{2}}, \mathbf{x}_{\mathbf{3}}\right\}$, we can not find a subset $\mathbf{V}_{\mathbf{Y}} \subseteq \mathbf{Y}\left(Z^{\mathbf{0}}=\emptyset\right)$ which 


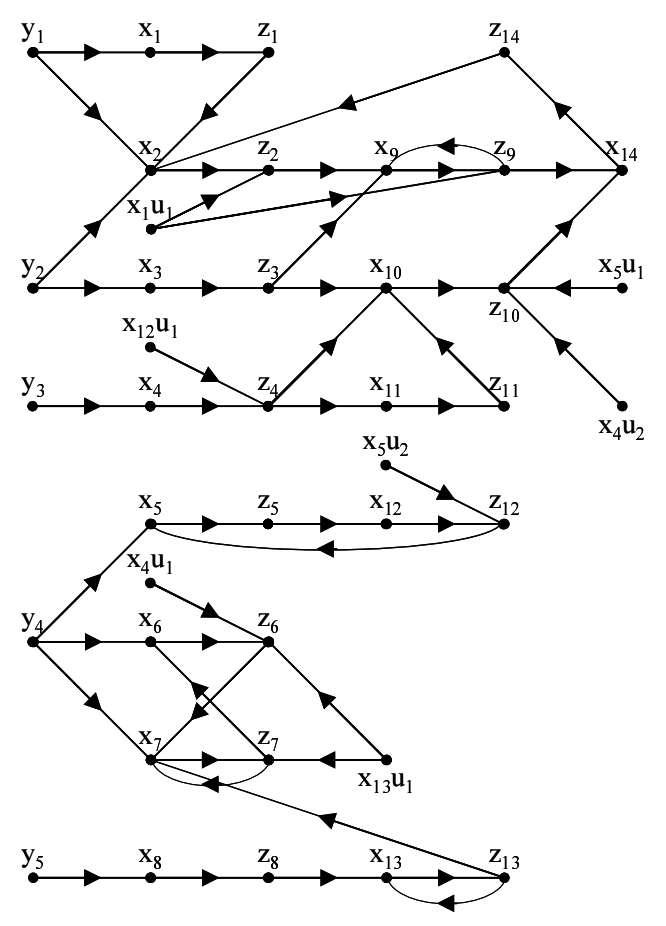

Figure 3: Digraph associated to Example 3
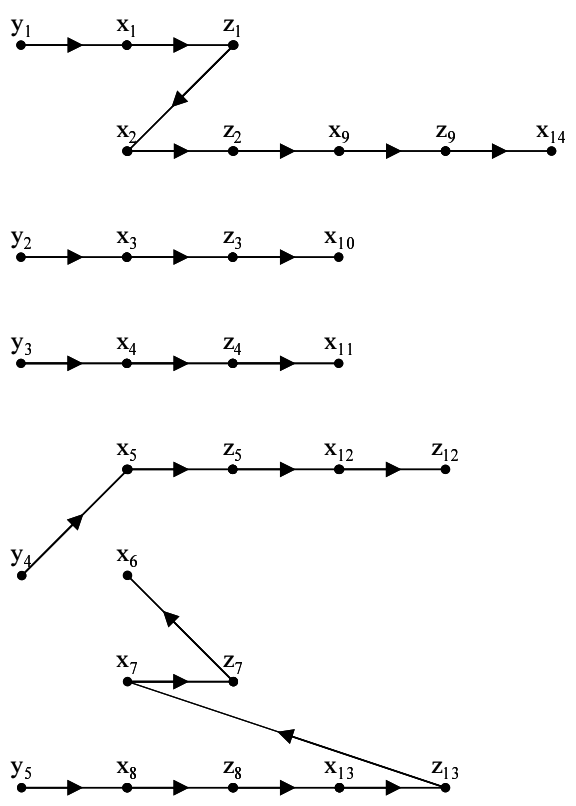

Figure 4: $v$-disjoint subgraph covering all the state vertices for Example 3 
allows to satisfy equality (Cond2) for $\mathbf{V}_{\kappa, \mathbf{j}}=\left\{\mathbf{z}_{\mathbf{1}}\right\},\left\{\mathbf{z}_{\mathbf{2}}\right\},\left\{\mathbf{z}_{\mathbf{3}}\right\},\left\{\mathbf{z}_{1}, \mathbf{z}_{\mathbf{2}}\right\}$ or $\left\{\mathbf{z}_{\mathbf{2}}, \mathbf{z}_{\mathbf{3}}\right\}$. Nevertheless, for $\mathbf{V}_{\mathbf{2}, \mathbf{2}}=\left\{\mathbf{z}_{\mathbf{1}}, \mathbf{z}_{\mathbf{3}}\right\}$ there exists $\mathbf{V}_{\mathbf{Y}}=\left\{\mathbf{y}_{\mathbf{1}}, \mathbf{y}_{\mathbf{2}}\right\}$, which allows to verify condition (Cond2): $\theta\left(\mathbf{V}_{\mathbf{Y}}\right)+\kappa+\operatorname{card}\left(\bar{\varphi}\left(\mathbf{V}_{\mathbf{2 , 2}}\right) \cap \mathbf{V}_{\mathbf{X U}^{\mathbf{0}}}^{*}\right)=2+2+0=4 \operatorname{and} \operatorname{card}\left(\varphi_{e}\left(\mathbf{V}_{\mathbf{Y}}\right) \cup \bar{\varphi}\left(\mathbf{V}_{\mathbf{2 , 1}}\right)\right)+1=3+1=4$.

Similarly, due to the presence of $\mathbf{x}_{\mathbf{1 2}} \mathbf{u}_{\mathbf{1}}$ in $\bar{\varphi}\left(\left\{\mathbf{z}_{\mathbf{4}}\right\}\right)$ and of $\mathbf{x}_{\mathbf{1 3}} \mathbf{u}_{\mathbf{1}}$ in $\bar{\varphi}\left(\left\{\mathbf{z}_{\mathbf{6}}\right\}\right) \bar{\varphi}\left(\left\{\mathbf{z}_{7}\right\}\right)$, there cannot exist a subset $\mathbf{V}_{\mathbf{Y}} \subseteq \mathbf{Y}$ which allows to satisfy equality (Cond2) for any nonempty subset $\mathbf{V}_{\kappa, \mathbf{j}} \subseteq\left\{\mathbf{z}_{\mathbf{4}}, \mathbf{z}_{\mathbf{5}}, \mathbf{z}_{\mathbf{6}}, \mathbf{z}_{7}\right\}$. Otherwise, for $\mathbf{V}_{\mathbf{1 , 8}}=\left\{\mathbf{z}_{\mathbf{8}}\right\}$, there exists $\mathbf{V}_{\mathbf{Y}}=\left\{\mathbf{y}_{\mathbf{5}}\right\}$, which allows to verify condition (Cond2): $\theta\left(\mathbf{V}_{\mathbf{Y}}\right)+\kappa+\operatorname{card}\left(\bar{\varphi}\left(\mathbf{V}_{\mathbf{1}, \mathbf{8}}\right) \cap \mathbf{V}_{\mathbf{X} \mathbf{U}^{\mathbf{0}}}^{*}\right)=1+1+0=2$ and $\operatorname{card}\left(\varphi_{e}\left(\mathbf{V}_{\mathbf{Y}}\right) \cup \bar{\varphi}\left(\mathbf{V}_{\mathbf{2}, \mathbf{1}}\right)\right)+1=1+1=2$. Thus, $\mathbf{Z}^{\mathbf{1}}=\left\{\mathbf{z}_{\mathbf{8}}, \mathbf{z}_{\mathbf{1}, \mathbf{3}}\right\}$. As $\theta\left(\mathbf{Z}^{\mathbf{1}}\right)=2>\theta\left(\mathbf{Z}^{\mathbf{0}}\right)=0$, we go to step 1 .

Step 1: $\quad$ Simple computations give $\mathbf{V}_{\mathbf{M}^{1}}^{*}=\left\{\mathbf{y}_{\mathbf{3}}, \mathbf{y}_{\mathbf{5}}, \mathbf{z}_{\mathbf{8}}\right\}, \quad \mathbf{V}_{\mathbf{X}^{1}}^{*}=\left\{\mathbf{x}_{\mathbf{4}}, \mathbf{x}_{\mathbf{8}}, \mathbf{x}_{13}\right\}, \quad \mathbf{V}_{\mathbf{X U}^{1}}^{*}=$ $\left\{\mathbf{x}_{\mathbf{4}} \mathbf{u}_{1}, \mathbf{x}_{\mathbf{4}} \mathbf{u}_{2}, \mathbf{x}_{13} \mathbf{u}_{\mathbf{1}}\right\}, \mathbf{Q}^{\mathbf{1}}=\varphi_{e}\left(\mathbf{Y} \cup \mathbf{Z}^{1}\right)=\left\{\mathbf{z}_{\mathbf{1}}, \mathbf{z}_{2}, \mathbf{z}_{\mathbf{3}}, \mathbf{z}_{\mathbf{4}}, \mathbf{z}_{5}, \mathbf{z}_{\mathbf{6}}, \mathbf{z}_{7}, \mathbf{z}_{\mathbf{8}}, \mathbf{z}_{\mathbf{9}}, \mathbf{z}_{10}, \mathbf{z}_{13}\right\}$.

For the same reasons than in step 0 , we cannot find subsets $\mathbf{V}_{\mathbf{Y}} \subseteq \mathbf{Y}$ and $\mathbf{V}_{\mathbf{Z}} \subseteq \wp\left(\mathbf{Z}^{\mathbf{1}}\right)$ which allow to satisfy equality (Cond2) for $\mathbf{V}_{\kappa, \mathbf{j}}=\left\{\mathbf{z}_{1}\right\},\left\{\mathbf{z}_{2}\right\},\left\{\mathbf{z}_{\mathbf{3}}\right\},\left\{\mathbf{z}_{\mathbf{1}}, \mathbf{z}_{\mathbf{2}}\right\},\left\{\mathbf{z}_{\mathbf{2}}, \mathbf{z}_{\mathbf{3}}\right\}$. Nevertheless, as previously, for $\mathbf{V}_{\mathbf{1 , 8}}=\left\{\mathbf{z}_{\mathbf{8}}\right\}$ and for $\mathbf{V}_{\mathbf{2 , 2}}=\left\{\mathbf{z}_{\mathbf{1}}, \mathbf{z}_{\mathbf{3}}\right\}$, there exist subsets $\mathbf{V}_{\mathbf{Y}} \subseteq \mathbf{Y}$ and $\mathbf{V}_{\mathbf{Z}} \subseteq \mathbf{Z}^{\mathbf{1}}$ which allow to satisfy equality (Cond2) and so $\mathbf{z}_{\mathbf{1 , 3}}$ and $\mathbf{z}_{\mathbf{8}}$ belong to $\mathbf{Z}^{\mathbf{2}}$. Besides, for $\mathbf{V}_{\mathbf{1}, \mathbf{1 3}}=\left\{\mathbf{z}_{\mathbf{1 3}}\right\}$, there exists $\mathbf{V}_{\mathbf{Y}}=\emptyset$ and $\mathbf{V}_{\mathbf{Z}}=\left\{\mathbf{z}_{\mathbf{8}}\right\} \subseteq \wp\left(\mathbf{Z}^{\mathbf{1}}\right)$ which allow to verify condition (Cond2): $\theta\left(\mathbf{V}_{\mathbf{Z}}\right)+\kappa+\operatorname{card}\left(\bar{\varphi}\left(\mathbf{V}_{\mathbf{1}, \mathbf{1 3}}\right) \cap \mathbf{V}_{\mathbf{X} \mathbf{U}^{1}}^{*}\right)=1+1+0=2 \operatorname{and} \operatorname{card}\left(\varphi_{e}\left(\mathbf{V}_{\mathbf{Z}}\right) \cup \bar{\varphi}\left(\mathbf{V}_{\mathbf{1 , 1 3}}\right)\right)+1=1+1=2$.

Furthermore, as $\mathbf{x}_{\mathbf{1 2}} \mathbf{u}_{\mathbf{1}} \in \bar{\varphi}\left(\left\{\mathbf{z}_{\mathbf{4}}\right\}\right)$, $\mathbf{x}_{\mathbf{1}} \mathbf{u}_{\mathbf{1}} \in \bar{\varphi}\left(\left\{\mathbf{z}_{\mathbf{9}}\right\}\right)$ and $\mathbf{x}_{\mathbf{5}} \mathbf{u}_{\mathbf{1}} \in \bar{\varphi}\left(\left\{\mathbf{z}_{\mathbf{1 0}}\right\}\right)$, no subset $\mathbf{V}_{\kappa, \mathbf{j}}$ containing $\mathbf{z}_{\mathbf{4}}, \mathbf{z}_{9}$ or $\mathbf{z}_{10}$ can satisfy (Cond1) and (Cond2). Finally, as $\mathbf{x}_{\mathbf{1 3}} \mathbf{u}_{\mathbf{1}}$ belongs to $\mathbf{V}_{\mathbf{X}}^{*}{ }^{1}$, for $\mathbf{V}_{\mathbf{3}, \mathbf{j}}=\left\{\mathbf{z}_{\mathbf{5}}, \mathbf{z}_{\mathbf{6}}, \mathbf{z}_{\mathbf{7}}\right\}$ there exist $\mathbf{V}_{\mathbf{Y}}=\left\{\mathbf{y}_{\mathbf{4}}\right\}$ and $\mathbf{V}_{\mathbf{Z}}=\emptyset$ which allow to verify condition (Cond2): $\theta\left(\mathbf{V}_{\mathbf{Y}}\right)+\kappa+\operatorname{card}\left(\bar{\varphi}\left(\mathbf{V}_{\mathbf{3}, \mathbf{j}}\right) \cap \mathbf{V}_{\mathbf{X \mathbf { U } ^ { 1 }}}^{*}\right)=1+3+2=6$ and $\operatorname{card}\left(\varphi_{e}\left(\mathbf{V}_{\mathbf{Y}}\right) \cup \bar{\varphi}\left(\mathbf{V}_{\mathbf{3}, \mathbf{j}}\right)\right)+1=5+1=6$. So, after step 1, we have $\mathbf{Z}^{\mathbf{2}}=\left\{\mathbf{z}_{\mathbf{8}}, \mathbf{z}_{\mathbf{1 3}}, \mathbf{z}_{\mathbf{1}, \mathbf{3}}, \mathbf{z}_{\mathbf{5}, \mathbf{6}, \mathbf{7}}\right\}$. As $\theta\left(\mathbf{Z}^{\mathbf{2}}\right)=4>\theta\left(\mathbf{Z}^{\mathbf{1}}\right)=1$, we go to step 2 .

Step 2: We have first compute $\mathbf{V}_{\mathbf{M}^{2}}^{*}=\left\{\mathbf{y}_{\mathbf{3}}, \mathbf{y}_{\mathbf{5}}, \mathbf{z}_{\mathbf{8}}, \mathbf{z}_{13}\right\}, \quad \mathbf{V}_{\mathbf{X}^{2}}^{*}=\left\{\mathbf{x}_{\mathbf{4}}, \mathbf{x}_{\mathbf{7}}, \mathbf{x}_{\mathbf{8}}, \mathbf{x}_{13}\right\}, \quad \mathbf{V}_{\mathbf{X U}^{2}}^{*}=$ $\left\{\mathbf{x}_{\mathbf{4}} \mathbf{u}_{\mathbf{1}}, \mathbf{x}_{\mathbf{4}} \mathbf{u}_{\mathbf{2}}, \mathbf{x}_{\mathbf{1 3}} \mathbf{u}_{\mathbf{1}}\right\}$. From the graph, we obtain $\mathbf{Q}^{\mathbf{2}}=\left\{\mathbf{z}_{\mathbf{1}}, \mathbf{z}_{\mathbf{2}}, \mathbf{z}_{\mathbf{3}}, \mathbf{z}_{\mathbf{4}}, \mathbf{z}_{\mathbf{5}}, \mathbf{z}_{\mathbf{6}}, \mathbf{z}_{\mathbf{7}}, \mathbf{z}_{\mathbf{8}}, \mathbf{z}_{\mathbf{9}}, \mathbf{z}_{\mathbf{1 0}}, \mathbf{z}_{\mathbf{1 2}}, \mathbf{z}_{13}\right\}$ Using the same arguments than for step 1 , we have that $\mathbf{z}_{\mathbf{8}}$ and $\mathbf{z}_{13}$ belongs to $\mathbf{Z}^{\mathbf{3}}$. As nothing changes for $\mathbf{z}_{\mathbf{1}}, \mathbf{z}_{\mathbf{2}}, \mathbf{z}_{\mathbf{3}}, \mathbf{z}_{\mathbf{4}}, \mathbf{z}_{\mathbf{9}}, \mathbf{z}_{10}$, only $\mathbf{z}_{\mathbf{1}, 3}$ belongs to $\mathbf{Z}^{\mathbf{3}}$. Otherwise, for $\mathbf{V}_{\mathbf{1}, 7}=\left\{\mathbf{z}_{7}\right\}$, there exist $\mathbf{V}_{\mathbf{Z}}=\left\{\mathbf{z}_{\mathbf{8}}, \mathbf{z}_{13}\right\} \subseteq \wp\left(\mathbf{Z}^{\mathbf{2}}\right)$ and $\mathbf{V}_{\mathbf{Y}}=\emptyset$ which allow to verify condition (Cond2): $\theta\left(\mathbf{V}_{\mathbf{Z}}\right)+\kappa+\operatorname{card}\left(\bar{\varphi}\left(\mathbf{V}_{\mathbf{2}, \mathbf{j}}\right) \cap \mathbf{V}_{\mathbf{X} \mathbf{U}^{2}}^{*}\right)=2+1+1=4$ and $\operatorname{card}\left(\varphi_{e}\left(\mathbf{V}_{\mathbf{Z}}\right) \cup \bar{\varphi}\left(\mathbf{V}_{\mathbf{1}, \mathbf{1 3}}\right)\right)+1=3+1=3$.

Furthermore, for $\mathbf{V}_{\mathbf{2}, \mathbf{j}}=\left\{\mathbf{z}_{\mathbf{5}}, \mathbf{z}_{\mathbf{6}}\right\}$, there exist $\mathbf{V}_{\mathbf{Y}}=\left\{\mathbf{y}_{\mathbf{4}}\right\} \subseteq \mathbf{Y}$ and a structurally minimal subset $\mathbf{V}_{\mathbf{Z}}=\left\{\mathbf{z}_{13}, \mathbf{z}_{\mathbf{8}}\right\} \subseteq \wp\left(\mathbf{Z}^{\mathbf{2}}\right)$ such that: $\theta\left(\mathbf{V}_{\mathbf{Y}} \cup \mathbf{V}_{\mathbf{Z}}\right)+\kappa+\operatorname{card}\left(\bar{\varphi}\left(\mathbf{V}_{\mathbf{1}, \mathbf{1 3}}\right) \cap \mathbf{V}_{\mathbf{X U}^{2}}^{*}\right)=3+2+2=7$ and $\operatorname{card}\left(\varphi_{e}\left(\mathbf{V}_{\mathbf{Y}} \cup \mathbf{V}_{\mathbf{Z}}\right) \cup \bar{\varphi}\left(\mathbf{V}_{\mathbf{1}, \mathbf{1 3}}\right)\right)+1=6+1=7$. The previous settings lead to $\mathbf{Z}^{\mathbf{3}}=\left\{\mathbf{z}_{\mathbf{7}}, \mathbf{z}_{\mathbf{8}}, \mathbf{z}_{\mathbf{1 3}}, \mathbf{z}_{\mathbf{1}, \mathbf{3}}, \mathbf{z}_{\mathbf{5}, \mathbf{6}}\right\}$ which is structurally minimal because of condition (Cond1). As $\theta\left(\mathbf{Z}^{3}\right)=5>\theta\left(\mathbf{Z}^{2}\right)=4$, we go to step 3 . 
Step 3: First, we compute $\mathbf{V}_{\mathbf{M}^{3}}^{*}=\left\{\mathbf{y}_{\mathbf{3}}, \mathbf{y}_{\mathbf{4}}, \mathbf{y}_{\mathbf{5}}, \mathbf{z}_{7}, \mathbf{z}_{\mathbf{8}}, \mathbf{z}_{13}, \mathbf{z}_{\mathbf{5}, \mathbf{6}}\right\}, \mathbf{V}_{\mathbf{X}^{3}}^{*}=\left\{\mathbf{x}_{\mathbf{4}}, \mathbf{x}_{\mathbf{5}}, \mathbf{x}_{\mathbf{6}}, \mathbf{x}_{\mathbf{7}}, \mathbf{x}_{\mathbf{8}}, \mathbf{x}_{12}, \mathbf{x}_{13}\right\}$, and $\mathbf{V}_{\mathbf{X U}^{3}}^{*}=\left\{\mathbf{x}_{\mathbf{4}} \mathbf{u}_{1}, \mathbf{x}_{\mathbf{4}} \mathbf{u}_{2}, \mathbf{x}_{5} \mathbf{u}_{1}, \mathbf{x}_{5} \mathbf{u}_{2}, \mathbf{x}_{12} \mathbf{u}_{1}, \mathbf{x}_{13} \mathbf{u}_{1}\right\}$. Graphically, we have $\mathbf{Q}^{\mathbf{3}}=$ $\left\{\mathbf{z}_{1}, \mathbf{z}_{2}, \mathbf{z}_{3}, \mathbf{z}_{4}, \mathbf{z}_{5}, \mathbf{z}_{\mathbf{6}}, \mathbf{z}_{7}, \mathbf{z}_{\mathbf{8}}, \mathbf{z}_{9}, \mathbf{z}_{10}, \mathbf{z}_{12}, \mathbf{z}_{13}\right\}$.

As $\mathbf{x}_{\mathbf{1 2}} \mathbf{u}_{\mathbf{1}} \in \mathbf{V}_{\mathbf{X U}}^{*}$, we have that $\mathbf{z}_{\mathbf{4}}$ belongs to $\mathbf{Z}^{\mathbf{4}}$ : for $\mathbf{V}_{\mathbf{1}, \mathbf{4}}=\left\{\mathbf{z}_{\mathbf{4}}\right\}$ and for $\mathbf{V}_{\mathbf{Y}}=\left\{\mathbf{y}_{\mathbf{4}}\right\}$, we have $\theta\left(\mathbf{V}_{\mathbf{Y}}\right)+\kappa+\operatorname{card}\left(\bar{\varphi}\left(\mathbf{V}_{\mathbf{1}, \mathbf{4}}\right) \cap \mathbf{V}_{\mathbf{X U}^{3}}^{*}\right)=1+1+1=3 \operatorname{and} \operatorname{card}\left(\varphi_{e}\left(\mathbf{V}_{\mathbf{Y}}\right) \cup \bar{\varphi}\left(\mathbf{V}_{\mathbf{1}, \mathbf{4}}\right)\right)+1=2+1=3$.

Moreover, the presence of $\mathbf{z}_{\mathbf{7}}$ in $\mathbf{Z}^{\mathbf{3}}$ implies that for $\mathbf{V}_{\mathbf{1 , 5}}=\left\{\mathbf{z}_{\mathbf{5}}\right\}$ there exist $\mathbf{V}_{\mathbf{Y}}=\left\{\mathbf{y}_{\mathbf{4}}\right\} \subseteq \mathbf{Y}$ and $\mathbf{V}_{\mathbf{Z}}=\left\{\mathbf{z}_{7}, \mathbf{z}_{13}, \mathbf{z}_{\mathbf{8}}\right\} \subseteq \wp\left(\mathbf{Z}^{\mathbf{3}}\right)$ such that condition (Cond2) is satisfied: $\theta\left(\mathbf{V}_{\mathbf{Y}} \cup \mathbf{V}_{\mathbf{Z}}\right)+\kappa+$ $\operatorname{card}\left(\bar{\varphi}\left(\mathbf{V}_{\mathbf{1 , 5}}\right) \cap \mathbf{V}_{\mathbf{X} \mathbf{U}^{3}}^{*}\right)=4+1+0=5$ and $\operatorname{card}\left(\varphi_{e}\left(\mathbf{V}_{\mathbf{Y}} \cup \mathbf{V}_{\mathbf{Z}}\right) \cup \bar{\varphi}\left(\mathbf{V}_{\mathbf{1}, \mathbf{5}}\right)\right)+1=4+1=5$.

Finally, due also to the presence of $\mathbf{z}_{\mathbf{7}}$ in $\mathbf{Z}^{\mathbf{3}}$ we have that for $\mathbf{V}_{\mathbf{1 , 6}}=\left\{\mathbf{z}_{\mathbf{6}}\right\}$ and for $\mathbf{V}_{\mathbf{Z}}=\left\{\mathbf{z}_{\mathbf{7}}, \mathbf{z}_{\mathbf{8}}, \mathbf{z}_{\mathbf{1 3}}\right\} \subseteq \wp\left(\mathbf{Z}^{\mathbf{3}}\right), \quad \theta\left(\mathbf{V}_{\mathbf{Z}}\right)+\kappa+\operatorname{card}\left(\bar{\varphi}\left(\mathbf{V}_{\mathbf{1 , 6}}\right) \cap \mathbf{V}_{\mathbf{X}}^{*} \mathbf{U}^{3}\right)=3+1+2=6$ and $\operatorname{card}\left(\varphi_{e}\left(\mathbf{V}_{\mathbf{Z}}\right) \cup \bar{\varphi}\left(\mathbf{V}_{\mathbf{1 , 6}}\right)\right)+1=5+1=6$.

Similarly, since $\mathbf{x}_{\mathbf{5}} \mathbf{u}_{\mathbf{1}} \in \mathbf{V}_{\mathbf{X}}^{*} \mathbf{U}^{3}$ we have that $\mathbf{z}_{\mathbf{1 2}}$ belongs to $\mathbf{Z}^{\mathbf{4}}$ : for $\mathbf{V}_{\mathbf{1 , 1 2}}=\left\{\mathbf{z}_{12}\right\}$ and for $\mathbf{V}_{\mathbf{Z}}=\left\{\mathbf{z}_{\mathbf{8}}, \mathbf{z}_{\mathbf{5}, \mathbf{6}}, \mathbf{z}_{\mathbf{1 3}}\right\} \subseteq \wp\left(\mathbf{Z}^{\mathbf{3}}\right)$, we have $\theta\left(\mathbf{V}_{\mathbf{Z}}\right)+\kappa+\operatorname{card}\left(\bar{\varphi}\left(\mathbf{V}_{\mathbf{1}, \mathbf{1 2}}\right) \cap \mathbf{V}_{\mathbf{X U}}^{*}\right)=3+1+1=5$ and $\operatorname{card}\left(\varphi_{e}\left(\mathbf{V}_{\mathbf{Z}}\right) \cup \bar{\varphi}\left(\mathbf{V}_{\mathbf{1}, \mathbf{4}}\right)\right)+1=4+1=5$. Therefore, we obtain $\mathbf{Z}^{\mathbf{4}}=\left\{\mathbf{z}_{\mathbf{4}}, \mathbf{z}_{\mathbf{5}}, \mathbf{z}_{\mathbf{6}}, \mathbf{z}_{\mathbf{7}}, \mathbf{z}_{\mathbf{8}}, \mathbf{z}_{\mathbf{1 2}}, \mathbf{z}_{\mathbf{1 3}}, \mathbf{z}_{\mathbf{1}, \mathbf{3}}\right\}$. As $\theta\left(\mathbf{Z}^{\mathbf{4}}\right)=7>\theta\left(\mathbf{Z}^{\mathbf{3}}\right)=5$, we go to step 4 .

Step 4: Firstly, we compute $\mathbf{V}_{\mathbf{M}^{4}}^{*}=\left\{\mathbf{y}_{\mathbf{3}}, \mathbf{y}_{\mathbf{4}}, \mathbf{y}_{\mathbf{5}}, \mathbf{z}_{\mathbf{5}}, \mathbf{z}_{\mathbf{6}}, \mathbf{z}_{7}, \mathbf{z}_{\mathbf{8}}\right\}, \mathbf{V}_{\mathbf{X}^{4}}^{*}=\left\{\mathbf{x}_{\mathbf{4}}, \mathbf{x}_{5}, \mathbf{x}_{\mathbf{6}}, \mathbf{x}_{7}, \mathbf{x}_{\mathbf{8}}, \mathbf{x}_{12}, \mathbf{x}_{13}\right\}$, $\mathbf{V}_{\mathbf{X U}^{4}}^{*}=\left\{\mathbf{x}_{\mathbf{4}} \mathbf{u}_{\mathbf{1}}, \mathbf{x}_{\mathbf{4}} \mathbf{u}_{\mathbf{2}}, \mathbf{x}_{5} \mathbf{u}_{\mathbf{1}}, \mathbf{x}_{5} \mathbf{u}_{\mathbf{2}}, \mathbf{x}_{\mathbf{1 2}} \mathbf{u}_{\mathbf{1}}, \mathbf{x}_{13} \mathbf{u}_{\mathbf{1}}\right\} . \quad$ We obtain graphically $\mathbf{Q}^{\mathbf{4}}=$ $\left\{\mathbf{z}_{1}, \mathbf{z}_{2}, \mathbf{z}_{3}, \mathbf{z}_{4}, \mathbf{z}_{5}, \mathbf{z}_{6}, \mathbf{z}_{7}, \mathbf{z}_{8}, \mathbf{z}_{9}, \mathbf{z}_{10}, \mathbf{z}_{11}, \mathbf{z}_{12}, \mathbf{z}_{13}\right\}$.

$\mathbf{z}_{\mathbf{4}} \in \mathbf{Z}^{\mathbf{4}}$ implies that for both $\mathbf{V}_{\mathbf{2}, \mathbf{j}}=\left\{\mathbf{z}_{\mathbf{1 0}}, \mathbf{z}_{\mathbf{1 1}}\right\}$ and $\mathbf{V}_{\mathbf{4}, \mathbf{j}^{\prime}}=\left\{\mathbf{z}_{\mathbf{1}}, \mathbf{z}_{\mathbf{2}}, \mathbf{z}_{\mathbf{9}}, \mathbf{z}_{10}\right\}$, there exist $\mathbf{V}_{\mathbf{Y}} \subseteq \mathbf{Y}$ and a structurally minimal vertex subset $\mathbf{V}_{\mathbf{Z}} \subseteq \wp\left(\mathbf{Z}^{\mathbf{i}}\right)$ such that (Cond2) is satisfied. Therefore, $\mathbf{Z}^{\mathbf{5}}=\left\{\mathbf{z}_{\mathbf{4}}, \mathbf{z}_{\mathbf{5}}, \mathbf{z}_{\mathbf{6}}, \mathbf{z}_{7}, \mathbf{z}_{\mathbf{8}}, \mathbf{z}_{12}, \mathbf{z}_{13}, \mathbf{z}_{1,3}, \mathbf{z}_{10,11}, \mathbf{z}_{1,2,9,10}\right\}$. As $\theta\left(\mathbf{Z}^{\mathbf{5}}\right)=9>\theta\left(\mathbf{Z}^{\mathbf{4}}\right)=7$, we go to step 5.

Step 5: As previously, $\mathbf{V}_{\mathbf{M}^{5}}^{*}=\left\{\mathbf{y}_{\mathbf{3}}, \mathbf{y}_{\mathbf{4}}, \mathbf{y}_{\mathbf{5}}, \mathbf{z}_{\mathbf{5}}, \mathbf{z}_{\mathbf{6}}, \mathbf{z}_{\mathbf{7}}, \mathbf{z}_{\mathbf{8}}\right\}, \mathbf{V}_{\mathbf{X}^{5}}^{*}=\left\{\mathbf{x}_{\mathbf{4}}, \mathbf{x}_{\mathbf{5}}, \mathbf{x}_{\mathbf{6}}, \mathbf{x}_{\mathbf{7}}, \mathbf{x}_{\mathbf{8}}, \mathbf{x}_{12}, \mathbf{x}_{13}\right\}$, $\mathbf{V}_{\mathbf{X} \mathbf{U}^{5}}^{*}=\left\{\mathbf{x}_{\mathbf{4}} \mathbf{u}_{1}, \mathbf{x}_{\mathbf{4}} \mathbf{u}_{\mathbf{2}}, \mathbf{x}_{\mathbf{5}} \mathbf{u}_{1}, \mathbf{x}_{\mathbf{5}} \mathbf{u}_{\mathbf{2}}, \mathbf{x}_{12} \mathbf{u}_{1}, \mathbf{x}_{13} \mathbf{u}_{1}\right\}$ and $\mathbf{Q}^{\mathbf{5}}=\mathbf{Z}$. Since $\mathbf{z}_{1,10,11} \in \mathbf{Z}^{\mathbf{5}}$ and $\mathbf{z}_{\mathbf{1}, 2,9,10} \in \mathbf{Z}^{\mathbf{5}}$, we can find $\mathbf{V}_{\mathbf{Y}} \subseteq \mathbf{Y}$ and a structurally minimal vertex subset $\mathbf{V}_{\mathbf{Z}} \subseteq \wp\left(\mathbf{Z}^{\mathbf{5}}\right)$ such that (Cond2) is satisfied for $\mathbf{V}_{\mathbf{2}, \mathbf{j}}=\left\{\mathbf{z}_{\mathbf{1}}, \mathbf{z}_{\mathbf{1 4}}\right\},\left\{\mathbf{z}_{\mathbf{1}}, \mathbf{z}_{\mathbf{3}}\right\},\left\{\mathbf{z}_{\mathbf{3}}, \mathbf{z}_{11}\right\}$ and $\left\{\mathbf{z}_{\mathbf{1 0}}, \mathbf{z}_{\mathbf{1 1}}\right\}$. Obviously it is also the case for any 2-subset of $\left\{\mathbf{z}_{\mathbf{1}}, \mathbf{z}_{\mathbf{3}}, \mathbf{z}_{\mathbf{1 0}}, \mathbf{z}_{\mathbf{1 1}}, \mathbf{z}_{\mathbf{1 4}}\right\}$, but we must choose only four of these 2-subsets to satisfy condition (Cond1) which ensures the structural minimality of $\mathbf{Z}^{\mathbf{6}}$. Moreover, we can also find $\mathbf{V}_{\mathbf{Y}} \subseteq \mathbf{Y}$ and a structurally minimal vertex subset $\mathbf{V}_{\mathbf{Z}} \subseteq \wp\left(\mathbf{Z}^{\mathbf{i}}\right)$ such that (Cond2) is satisfied for $\mathbf{V}_{\mathbf{3}, \mathbf{j}}=\left\{\mathbf{z}_{1}, \mathbf{z}_{2}, \mathbf{z}_{\mathbf{9}}\right\}$. Consequently, $\mathbf{Z}^{\mathbf{6}}=\left\{\mathbf{z}_{\mathbf{4}}, \mathbf{z}_{5}, \mathbf{z}_{\mathbf{6}}, \mathbf{z}_{7}, \mathbf{z}_{\mathbf{8}}, \mathbf{z}_{12}, \mathbf{z}_{13}, \mathbf{z}_{1,3}, \mathbf{z}_{1,14}, \mathbf{z}_{3,11}, \mathbf{z}_{10,11}, \mathbf{z}_{1,2,9}\right\}$. As $\theta\left(\mathbf{Z}^{\mathbf{6}}\right)=10>\theta\left(\mathbf{Z}^{\mathbf{5}}\right)=9$, we go to step 6 .

Step 6: $\quad$ We compute $\mathbf{V}_{\mathbf{M}^{6}}^{*}=\left\{\mathbf{y}_{\mathbf{1}}, \mathbf{y}_{\mathbf{2}}, \mathbf{y}_{\mathbf{3}}, \mathbf{y}_{\mathbf{4}}, \mathbf{y}_{\mathbf{5}}, \mathbf{z}_{\mathbf{4}}, \mathbf{z}_{\mathbf{5}}, \mathbf{z}_{\mathbf{6}}, \mathbf{z}_{\mathbf{7}}, \mathbf{z}_{\mathbf{8}}, \mathbf{z}_{1,14}, \mathbf{z}_{1,3}, \mathbf{z}_{3,11}, \mathbf{z}_{10,11}\right\}$ and as $\mathbf{V}_{\mathbf{X}^{6}}^{*}=\mathbf{X}$, we can easily deduce that $\mathbf{Z}^{7}=\mathbf{Z}=\mathbf{Z}^{*}$. According to Condition (2) of Corollary 1, we can conclude to the generic uniform observability of the system. 


\section{Conclusion}

In this paper, we propose an analysis tool to study the generic uniform observability of structured bilinear systems. Using a graphic representation dedicated to this class of nonlinear systems two conditions are provided and expressed in graphic terms. The first one is a necessary condition and can be checked directly from the digraph associated to the system. The second one is sufficient and necessitates the use of an iterative algorithm providing the computation of a particular vertex subset. The proposed conditions, which need few information about the system, are very easy to check by means of well-known combinatorial techniques and simply by hand for small systems. That makes our approach particularly suited for large-scale and sparse systems as it is free from numerical difficulties. Indeed, the proposed algorithm and conditions can be easily implemented because they require simple computations on integers and are based on finding edges and subgraphs in a digraph.

Starting from the presented results, we can study, as in [5] for linear systems, the optimisation of the sensor location to achieve the generic uniform observability. Finally, sensor defects and also the addition of new actuators to the system, for fault tolerant control strategy for example, can lead to the lost of the generic uniform observability property. One of the perspectives of the presented work can be also the study of the actuator placement and sensor redundancy in order to preserve the generic uniform observability property.

\section{References}

[1] G. Bornard and H. Hammouri. A graph approach to uniform observability of linear multi output systems. In $41^{\text {st }}$ IEEE Conference on Decision and Control, pages:701-706, Las Vegas, U.S.A., 2002.

[2] T. Boukhobza, M. Djemai and J-P. Barbot. Implicit triangular observer form dedicated to a sliding mode observer for systems with unknown inputs. Asian Journal of Control, 5(4):513-527, 2004.

[3] V. N. Brandin, Y. M. L. Kostyukovskii and G. N. Razorenov. Global observability conditions for nonlinear dynamic systems. Automation and Remote Control, 36(10):18-25, 1975.

[4] C. Bruni, G. Di Pillo and G. Koch. Bilinear systems: An appealing class of nearly linear systems in theory and application. IEEE Transactions on Automatic Control, AC-19(4):334-348, 1974.

[5] C. Commault, J-M. Dion and D. H. Trinh Observability recovering by additional sensor implementation in linear structured systems. Proceedings of the 44th IEEE Conference on Decision and Control, and the European Control Conference, Seville, Spain. 2005.

[6] E. J. Davison and S. H. Wang. Properties of linear time-invariant multivariable systems subject to arbitrary output and state feedback. IEEE Transactions on Automatic Control, AC-18(1):24-32, 1973. 
[7] F. Deza, E. Busvelle, J-P. Gauthier and D. Rakotopara. High gain estimation for nonlinear systems. System E Control Letters, 18(4):295-299, 1992.

[8] J-M. Dion, C. Commault and J. Van der Woude. Generic properties and control of linear structured systems: A survey. Automatica, 39(7):1125-1144, 2003.

[9] J-P. Gauthier and G. Bornard. Observability for any $u(t)$ of a class of nonlinear systems. IEEE Transactions on Automatic Control, 26(4):922-926, 1981.

[10] J-P. Gauthier, H. Hammouri and S. Othman. A simple observer for nonlinear systems: Application to bioreactors. IEEE Transactions on Automatic Control, 37(6):875-880, 1992.

[11] J-P. Gauthier and I. A. K. Kupka. Observability and observers for nonlinear systems. SIAM Journal of Control and Optimization, 32(4):975-994, 1994.

[12] K. Glover and L. M. Silverman. Characterization of structural controllability approach. IEEE Transactions on Automatic Control, AC-21(4):534-537, 1976.

[13] R. Hermann and A. J. Krener. Nonlinear controllability and observability. IEEE Transactions on Automatic Control, AC-22(5):728-740, 1977.

[14] M. Hou, K. Busawon and M. Saif. Observer design based on triangular form generated by injective map. IEEE Transactions on Automatic Control, 45(7):1350-1355, 2000.

[15] S. R. Kou, D. L. Elliot and T. J. Tarn. Observability of nonlinear systems. Information and Control, 22(1):89-99, 1973.

[16] R. R. Mohler and W. Kolodziej. An overview of bilinear system theory and applications. IEEE Transactions on System, Man and Cybernetics, SMC-10(10):683-688, 1980.

[17] K. Murota. System Analysis by Graphs and Matroids. Springer-Verlag, New York, U.S.A., 1987.

[18] K. J. Reinschke. Multivariable Control. A Graph Theoretic Approach. Springer-Verlag, New York, U.S.A., 1988.

[19] J. Rudolph and M. Zeitz. A block triangular nonlinear observer normal form. System E Control Letters, 23(1):1-8, 1994.

[20] E. D. Sontag. Mathematical control theory, volume 6 of Texts in Applied Mathematics. SpringerVerlag, New York, Berlin, Heidelberg, 2nd edition, 1998.

[21] H. J. Sussmann. Single input observability of continuous time systems. Mathematical System Theory, 12(4):371-393, 1979.

[22] F. Svaricek. A graph theoretic approach for the investigation of the observability of bilinear systems. In Proceedings of the 12th IFAC World Congress, pages:351-354, Sydney, Australia, 1993.

[23] J. Van der Woude. A graph theoretic characterization for the rank of the transfer matrix of a structured system. Mathematics of Control, Signals and Systems, 4(1):33-40, 1991.

[24] D. Williamson. Observation of bilinear systems with application to biological control. Automatica, 13(3):243-254, 1977. 